\title{
ABSTRACTS
}

\section{Abstracts from the 5th International Porto Congress of Multiple Sclerosis}

Maria José Sá

(c) The Author(s) 2019

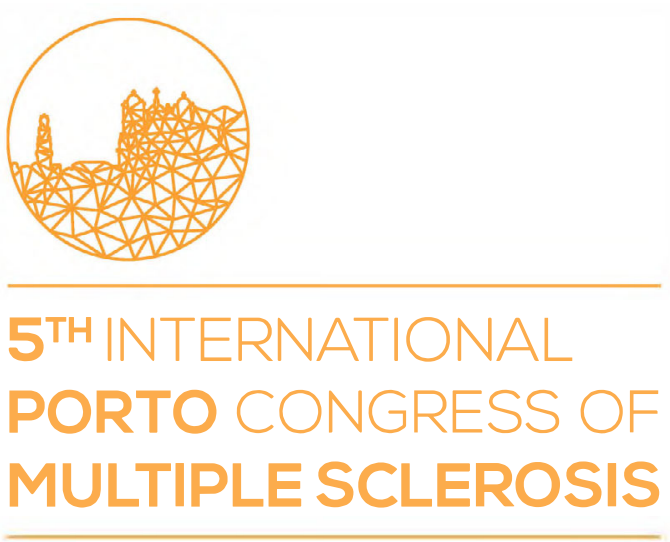

\author{
FEBRUARY 14 - 16, 2019 \\ PORTO - PORTUGAL \\ ORDEM DOS MÉDICOS
}

BACK TO EXACT SCIENCES: BRIDGING

SOLUTIONS FOR GLOBAL MS CHALLENGES

Enhanced Digital Features To view enhanced digital features for this article go to https://doi.org/10.6084/ m9.figshare.7925261.

M. J. Sá $(\bowtie)$

Department of Neurology, Hospital de S. Joáo Al.

Hernáni Monteiro, Porto, Portugal

e-mail: mjsa@med.up.pt

M. J. Sá

Faculty of Health Sciences, University Fernando

Pessoa, Rua Carlos da Maia, Porto, Portugal 


\section{INTRODUCTION}

The 5th International Congress of Multiple Sclerosis was once more held in Porto, from February 14th to 16th, 2019. In line with the rapid development of science, technology and lifestyles, the 2019 Congress focused on the encounter between basic and clinical sciences. The intensive program covered a wide range of topics including the most recent advances regarding demyelinating diseases, their underlying molecular mechanisms, treatment advances and a broad view of the management of multiple sclerosis (MS) worldwide. The latest findings and updates on these key issues were discussed by leading experts in the field in four oral sessions entitled 'MS in the World, the World of MS', 'Maths and Physics in MS', 'Hot Topics Grand Round', and 'From Bench to Bedside'. Furthermore, the selection of oral and poster presentations included in the current supplementary issue of Neurology and Therapy provided a forum for attendees to share, debate and analyze the most recent advances about the etiopathogenesis, therapeutic innovations, unmet needs and future challenges in the area of MS.

EPOSTERS ABSTRACTS

\section{EPIOO1}

Evaluation of Persistent Fatigue in Multiple Sclerosis Patient with Low Disability

Larissa Garcia ${ }^{1}$; Regina Alvarenga ${ }^{1}$; Cleonice Bento ${ }^{1}$; Helcio Alvarenga Filho ${ }^{1}$

${ }^{1}$ Universidade Federal Do Estado Do Rio De Janeiro

Introduction: Fatigue in multiple sclerosis (MS) is primary and is aggravated by secondary causes and environmental factors. Several cross-sectional studies with application of the fatigue severity scale (FSS) in MS confirm the high frequency of fatigue in this disease, regardless of disability. Persistent fatigue has a lack of studies, because it requires studies with longitudinal design. A new fatigue scale for MS was recently validated for the Portuguese language, NFISMS/BR.

Objectives: To evaluate subjective fatigue in a longitudinal study in MS patients applying two scales. Methodology: Patients attended consecutively at Hospital da Lagoa (Rio de Janeiro) between April and June 2017 with relapsing-remitting MS, EDSS from zero to 2.5 , out of the relapse and without comorbidities were invited to participate in the study.
Clinical data were collected in medical records. The questionnaires of the FSS and NFIS-MS/BR scales were completed in three interviews with a three-month interval. Persistent fatigue was defined by fatigue scores in FSS $(\geq 28)$ or NFI-MS/BR $(\geq 30)$ in the three collections and sporadic fatigue in at least one of these collections.

Results and Conclusions: The total of 38 patients who answered the scales in the first interview, fatigue was identified in $74.4 \%$ by FSS and $64 \%$ by NFIS-MS/BR. Twenty-six patients who attended the three interviews, fatigue by FSS was persistent in $54 \%$ of the patients, sporadic in $38 \%$ and $8 \%$ without fatigue. According to NFI-MS/BR fatigue was persistent in $47 \%$ of the patients, sporadic in $33 \%$ and $20 \%$ without the symptom. Conclusion: Fatigue occurs in the majority of patients with MS with mild disability, being persistent in about $50 \%$ of the cases. FSS showed greater sensitivity for fatigue identification, but the NFIS-MS/BR presents items that best describes the characteristics of fatigue in MS.

Funding: This research was sponsored by Pesquisa de Produtividade Universidade Estacio de Sá.

Keywords: Multiple sclerosis; Fatigue

\section{EPIOO2}

Quality of Life of the Person with Multiple Sclerosis [1]

Rosa Martins ${ }^{1}$

${ }^{1}$ Instituto Politécnico de Viseu

Introduction: Multiple sclerosis (MS) is an inflammatory, demyelinating and unpredictable disease, which its origin and treatment is yet unknown. It is the chronic neurological disease that more affects young adults, at working age, and might develop into situations of varying levels of disability.

Objectives: This study's main objective was to understand the perception of quality of life (QOL), from vulnerability to stress and social support of people with MS, as well as the socio- demographic, clinical and psychosocial influences.

Methodology: It was conducted a cross-sectional, descriptive-correlational study, of quantitative nature, where $54 \mathrm{MS}$ patients attended, most of them were women $(61.1 \%)$, married $(72.2 \%)$, with an age average of 42 years, employed (37.0\%), with an age average of 33 years old at the beginning of the disease. To measure the variable the following instruments were used: Social-Demographic/Clinic Questionnaire, Multiple Sclerosis Scale and Quality of 
Life, Barthel Index, Scale of Vulnerability to Stress and Social Support Scale.

Results and Conclusions: The MS patients between 20 and 31 years, employed, with younger age at the beginning of the disease, without sequelae and with reduced level of dependence have better physical functioning; the patients that have associated disease are the one's who report a greater bodily pain and a better cognitive functioning; the one's that show higher functional capacity have better social skills; the one's who carry out rehabilitation have better mental health; MS patients between 56 and 67 years with a higher age at the beginning of the disease are the one's who manifest worse sexual functioning; the one's who have better general QOL are those who are satisfied with their jobs. There are also significant influences between QOL and all factors of vulnerability to stress, as well as between QOL and all factors of social support (in relation to psychosocial variables).

Funding: No funding or sponsorship was received for this research.

Keywords: Multiple sclerosis; Quality of life; Dependence degree; Stress; Social support

\section{EPIOO3}

Health-Related Quality of Life in Patients with Multiple Sclerosis without Disability

Helcio Alvarenga Filho ${ }^{1,2}$; Mariana Santos ${ }^{2}$; Cleonice Bento $^{2}$; Anke Bergmann ${ }^{3}$

${ }^{1}$ Universidade Estácio De Sá; ${ }^{2}$ Universidade Federal Do Estado Do Rio De Janeiro; ${ }^{3}$ Inca

Introduction: Studies on health-related quality of life (HRQoL) describe a reduction in all Short Form (SF)36 scores in patients with multiple sclerosis (MS) with different levels of disability.

Objectives: Here we analysed HRQoL in patients with MS without disability and compared it with that in healthy controls, as well as investigated the impact of subclinical depression and fatigue in the physical and mental domains.

Methodology: This is a cross-sectional study of Brazilian patients from Rio de Janeiro with relapsing-remitting MS who had Extended Disability Status Scale scores of $0-1.5$. Instruments used included SF36 to assess HRQoL, Beck Depression Inventory to assess depression, Fatigue Severity Scale and 6-minute walk test to assess ambulation and Paced Auditory Serial Addition Task to assess cognition, and the results were compared between patients with MS and healthy controls. Multiple linear regression models were applied in each of the eight SF-36 domains to analyse the influence of demographic and clinical factors on the reduction of HRQoL.

Results and Conclusions: Fifty-four individuals were included, 31 with an MS diagnosis and 23 healthy controls. There were no significant differences in demographic and clinical characteristics except for the presence of fatigue (MS: $64.5 \%$ vs. control: $21.7 \%$, $p=0.002$ ). Sedentary lifestyle, depression, fatigue and MS diagnosis had a negative impact on specific SF-36 scores. However, in the multiple regression model, only depression and fatigue were associated with reduced SF-36 scores. Patients with depression had worse scores for physical function $(p=0.006)$, bodily pain $(p=0.011)$, vitality $(p<0.001)$, roleemotional $(p<0.001)$ and mental health $(p<0.001)$, whereas patients with fatigue had worse scores for physical function $(p=0.006)$ and vitality $(p=0.001)$. Conclusions: MS without disability does not affect HRQoL. Fatigue and depression in MS patients or healthy controls, independently affects HRQoL, reducing specific SF-36 scores, particularly vitality.

Funding: This research was sponsored by Coordenação de Aperfeiçoamento de Pessoal de Nível Superior (CAPES)

Keywords: Multiple sclerosis; Quality of life; Fatigue; Depression

\section{EPIOO4}

Association Between Kappa Index and Disease Severity in Multiple Sclerosis

Paloma Menendez-Valladares ${ }^{1}$; Maria Isabel GarciaSánchez ${ }^{2}$; Sónia Estêvão Neto ${ }^{3}$; Carmen Bermudo Guitarte ${ }^{1}$

${ }^{1}$ Unidad de Gestión Clínica Bioquímica Clínica, Hospital Universitario Virgen Macarena, Spain; ${ }^{2}$ Biobanco Hospitalario Virgen Macarena, Biobanco del Sistema Sanitario Público de Andalucía, Spain; ${ }^{3}$ The Binding Site-Portugal

Introduction: The Expanded Disability Status Scale (EDSS) is a widely used instrument to quantify clinical severity and functional deficits in multiple sclerosis (MS). The EDSS consists in an ordinal rating system ranging from 0 (normal) to 10 (death).

Objectives: Analyze the correlation between kappa index and EDSS at the beginning and at the end of the study, in MS patients.

Methodology: 106 MS patients, diagnosed according with the McDonald criteria (2010), were included in this study. The EDSS and the kappa index were determined at the beginning of the study and after 2-year follow-up.

Free Light Chains Kappa (FLCk) were measured by nephelometry in cerebrospinal fluid (CSF) samples. Kappa index was calculated as [(FLCk_CSFxAlbumin_serum)/(FLCk_serum)xAlbumin_CSF)]. 
Results and Conclusions: Comparison of EDSS and kappa index at the diagnosis showed no correlation $(p=0.175)$. The median values of Kappa index progressively increased when dividing patients in four groups according to the EDSS at baseline (kappa index median: group 1 (EDSS $<2) 20.82$, group 2 $($ EDSS $=2-2.5)$ 56.65, group 3 (EDSS $=3-3.5) 62.33$, group 4 (EDSS $\geq 4$ ) 79.23). Grouping these patients according to a kappa index cut-off $=10.62$, established in previous studies, showed statistically significant differences $(p=0.028)$. Baseline EDSS $\geq 2$ was found in $67.47 \%$ of patients with kappa index $>10.62$. While only $39.89 \%$ of patients with kappa index $<10.62$ showed baseline EDSS $\geq 2$.

On the other hand, comparing EDSS at 2-years follow-up versus baseline kappa index, showed no statistically significant correlation $(p=0.770)$. Neither grouping patients in four groups according to EDSS at two-years and kappa index cut-off of 10.62 $(p=0.319)$.

These results suggest an association between high kappa index values and a higher disability status of patients at the beginning, supporting the hypothesis of the presence of FLCk intrathecal synthesis in MS pathophysiological process.

The EDSS at 2-years follow-up must be affected by other factors, such as MS treatment.

Funding: No funding or sponsorship was received for this research.

Keywords: Multiple sclerosis (MS); Kappa Index; Expanded Disability Status Scale (EDSS)

\section{EPIOO5}

Multiple Sclerosis and Vitamin D: Case Control Study Made in the Latitude-22

Fernanda Ferreira Chaves Costa Pereira ${ }^{1}$; Ana Beatriz Calmon Pereira $^{1,2}$; Claudia Cristina Ferreira Vasconcelos ${ }^{1}$; Regina Maria Papais Alvarenga ${ }^{1}$; Oscar Fernandez ${ }^{3}$

${ }^{1}$ Universidade Federal do Estado do Rio de Janeiro, UNIRIO Brasil; ${ }^{2}$ Universidade de Vassouras; ${ }^{3}$ Hospital Regional Universitario Carlos Haya-Malaga

Introduction: The peculiar geographical distribution of multiple sclerosis (MS) suggests the presence of an environmental factor in the genesis of this disease. It has been proposed for decades that vitamin D deficiency could be a possible explanation for the latitudinal gradient of MS.

Objectives: To evaluate vitamin D levels in patients with multiple sclerosis in the Fluminense Southern Region of Brazil.
Methodology: This is a cross-sectional case control study of a cohort in follow-up over the last 10 years. Results and Conclusions: Results: Data from 50 patients and 50 controls were obtained. The female sex was more frequent. The mean age of the two groups is found in the 5th decade of life. In the group of patients the median EDSS was 2, ranging from 0 to 8.5 and the average disease time was 8.7 years ( \pm 7.5 years). There was no significant difference between the mean serum levels of vitamin $D$ between the groups studied. However, the majority of participants in both groups had serum levels of vitamin D below normality. There was also no difference between these two groups when assessing the time of daily exposure to sun, season of birth and vitamin D status. Conclusion: In Brazil there is great availability of solar radiation. Despite this, the majority of study participants had serum levels of vitamin D below those considered normal. Further studies are needed in different populations for greater conclusions.

Funding: This research was sponsored by CAPES (Coordenação de Aperfeiçoamento de Pessoal de Nivel Superior).

Keywords: Vitamin D; Multiple sclerosis; Latitude; Brazil; Case control

\section{EPIO06}

Epidemiological Features and Study of Risk Factors for Clinical Conversion from RIS To MS in the City of Rio De Janeiro

Rafaella Eliria Ericksson ${ }^{1}$; Nadja Emidio De Araujo ${ }^{1}$; Marcos Papais Alvarenga ${ }^{1}$; Claudia Cristina F. Vasconcelos ${ }^{1}$; Regina Maria Papais Alvarenga ${ }^{1}$; Helcio Alvarenga Filho ${ }^{1,2}$; Vanderson Carvalho Neri ${ }^{1}$

${ }^{1}$ Unirio, Hospital Da Lagoa; ${ }^{2}$ UNESA

Introduction: Radiologically isolated syndrome (RIS) is an uncommon condition characterized by incidental findings on central nervous system, highly suggestive of demyelination disease, on a magnetic resonance imaging (MRI) in healthy individuals, without complaints and normal neurological examination. Most of the studies considered RIS preclinical phase of multiple sclerosis (MS), even though no studies to support this concept yet. Studies are aimed to define a guideline for this management. In Brazil, only one article was published data in this subject.

Objectives: Report cases of RIS, describing demographic, clinical, and laboratory characteristics, and identify possible predictors of risk for clinical conversion. 
Methodology: In a retrospective descriptive study, eight cases with diagnosis of RIS by Okuda criteria 2009, attended from 2009 to 2013 at reference center for MS in Rio de Janeiro, Brazil, were screened for this study.

Results and Conclusions: Four cases (50\%) converted to MS in periods between 10 months to 8 years; two cases (25\%) presented new lesions and maintaining asymptomatic; and two cases (25\%) there was no evolution. Caucasian (62.5\%) and female sex (87.5\%) are the highest prevalence. The mean age at first examination was 32.4 years (8-53). Headache was the more common cause for the first imaging exam (37.5\%). Sensory symptoms were the initial clinical manifestation in all cases of conversion. The predictor of risk to conversion in this group were infratentorial and or spinal cord lesions in the first exam and the presence of more than nine lesions typical of MS associated with changes in cerebrospinal fluid (CSF), such as oligoclonal bands or increased G-type immunoglobulins. Conclusion: The presence of infratentorial lesions at the first exam, and more than nine lesions with CSF abnormal may be associated with a higher risk to clinical conversion.

Funding: No funding or sponsorship was received for this research.

Keywords: Radiologically isolated syndrome; Multiple sclerosis; Magnetic resonance imaging.

\section{EPIOO7}

\section{Alterations on Circulating T Cell Functional Compartments in Relapsing-Remitting Multiple Sclerosis Patients}

Andreia Monteiro ${ }^{1,2}$; Catarina Cruto $^{3}$; Pedro Rosado ${ }^{3}$; Luiza Rosado $^{3}$; Ana Mafalda Fonseca ${ }^{1}$; Artur Paiva ${ }^{4,5,6}$

${ }^{1}$ Centro de Investigação em Ciências da Saúde, Universidade Beira Interior (CICS-UBI); ${ }^{2}$ Serviço Patologia Clínica, Centro Hospitalar Universitário Cova da Beira; ${ }^{3}$ Serviço Neurologia, Centro Hospitalar Universitário Cova da Beira; ${ }^{4}$ Unidade de Gestão Operacional de Citometria, Serviço de Patologia Clínica, CHUC; ${ }^{5}$ Coimbra Institute for Clinical and Biomedical Research (iCBR), Faculdade de Medicina; Universidade de Coimbra; ${ }^{6}$ Escola Superior de Tecnologia da Saúde de Coimbra

Introduction: Currently, the pathogenic potential of Th17 pathways, in addition to those from Th1, in the development of MS has been widely accepted, although not fully understood.

Objectives: In this context, we aimed to quantify and functional characterize circulating Th1, Th2, Th17, Treg, Tc1, Tc2, Tc17 cells and the serum level of IL-17 from patients diagnosed with relapsing-remitting MS (RRMS).
Methodology: 38 patients with RRMS treated with IFNb, 30 in remission and 8 in relapse were included in this study, as well as, 20 healthy age- and gendermatched volunteers.

The Th1/c1 and Th2/c2 subsets were defined based on the differential expression of CCR5 and CCR4 and the functional characterization was evaluated by intracellular cytokine (IL-17, TNF $\alpha$, IFN $\gamma$ and IL-2) staining after stimulation with PMA + Ionomycin, both assessed by flow cytometry. The results were analyzed using Infinicyt software. Mann-Whitney $U$ test (average \pm standard deviation; median) was used for statistical evaluation. The serum level of IL17 was measured by ELISA.

Results and Conclusions: We observed a significantly decrease in the ratio Th1/Th2 $\left(\mathrm{CCR} 5^{+} / \mathrm{CCR} 4^{+}\right)$in the remission group when compared with the relapse patients and with the healthy group (HG). Moreover, we found a decreased frequency of $\mathrm{CD}^{+} \mathrm{T}$ cells producing IFN $\gamma$ and IL-2 in remission MS patients and a decrease of $\mathrm{CD}^{+} \mathrm{T}$ cells producing IFN $\gamma$ in relapse. Concerning $\mathrm{T}$ cells producing IL-17, our results pointed to an increased frequency of Th17 and Tc17 cells in remission group. Among Th17 cells we observed a decreased frequency of those producing IFN $\gamma$ in the relapse group. The serum levels of IL-17 increased in both phases of MS. Taken together our results pointed to significant changes in circulating $T$ cell homeostasis from MS patients, some of them correlating with the disease phase, namely an increase of $\mathrm{CCR}^{+} / \mathrm{CD} 4^{+}(\mathrm{Th} 2) \mathrm{T}$ cells and of Th17 and Tc17 subpopulations in remission patients.

Funding: No funding or sponsorship was received for this research.

Keywords: T cell; Relapsing-remitting; Periphery

\section{EPIOO8}

Diverse Gut Microbiota Composition in Portuguese Multiple Sclerosis Patients

Amélia Sarmento ${ }^{1,2}$; Daniela Ferro ${ }^{3}$; Liliana Mendonça ${ }^{3}$; Gara Molina $^{4}$; Carles Ubeda ${ }^{4}$; Maria José Sá ${ }^{1,3}$

${ }^{1}$ FP-ENAS (UFP Energy, Environment and Health Research Unit), CEBIMED (Biomedical Research Centre), University Fernando Pessoa, Porto, Portugal; ${ }^{2}$ Instituto de Investigação e Inovação em Saúde, IBMC, Instituto de Biologia Molecular e Celular, Universidade do Porto, Portugal; ${ }^{3} \mathrm{MS}$ Clinic, Department of Neurology, Centro Hospitalar Universitário São João, Porto, Portugal; ${ }^{4}$ Departamento de Genómica y Salud, Centro Superior de Investigación en Salud Pública, FISABIO

Introduction: The influence of gut microbiota in host immune and inflammatory status has been pointed out as an important predisposing factor in multiple sclerosis (MS) and other inflammatory diseases. 
Objectives: In this work, we investigated the differences in the gut microbiota of a cohort of Portuguese MS patients and healthy controls.

Methodology: MS patients $(n=27)$ and HC $(n=26)$ were recruited from the MS Clinic of our Hospital. Feces were collected and DNA extracted using a commercial kit. The V3-V4 regions of the 16S rRNA gene were amplified and sequenced using the MiSeq platform from Illumina. Amplicon sequence variants (ASVs) were identified with DADA2. Taxonomic composition was assigned using the Silva database. Wilcoxon test and False Discovery Rate were applied to identified microbiota differences (i.e. genera, ASVs, Shannon diversity).

Results and Conclusions: The richness (number of ASVs) and diversity (Shannon index) of the gut microbiota were similar between MS patients and HC. The overall microbiota structure was different among HC and MS patients at the genus and ASV levels (Adonis; $p=0.032$ and $p=0.053$, respectively). Seven genera were significantly decreased in MS patients $(p<0.05$; FDR $<0.2)$ : Turicibacter, Oscillospira, Terrisporobacter, Clostridium, Lachnospiraceae_ND3007, Intestinibacter and Fusicatenibacter. Three genera were increased: Victivallis, Gordonibacter and Eggerthella $(p<0.05 ;$ FDR $<0.2)$. A decrease in ASVs belonging to the genera Clostridium, Subdoligranulum and Lachnospiraceae and an increase in one ASV belonging to Alistipes finegoldii was identified in MS patients $(p<0.05 ;$ FDR $<0.2)$. In addition, we found a decrease in ASVs belonging to the Faecalibacterium prausnitzii and Fusicatenibacter saccharivorans species $(p<0.05$; FDR $<0.27)$, which has also been reported in other inflammatory diseases.

The decrease we found in Faecalibacterium prausnitzii and in Lachnospiraceae, as well as the increase in Actinobacteria (Gordonibacter, Eggerthella) is in accordance to what has been described for patients with MS. Analysis of possible correlations between clinical or immunological data and microbiota composition will further be performed.

Funding: This research was sponsored by the Merck Grant: "Intestinal microbiota in multiple sclerosis and its impact in the development of inflammation". Keywords: Multiple sclerosis; Gut; Microbiota

\section{EPIO09}

\section{Spinal Form of Asian Multiple Sclerosis in Brazil}

Rafaella Eliria Ericksson ${ }^{1}$; Jonath Tigre ${ }^{1}$; Anna Christiany Brandão ${ }^{1}$; Vanderson Neri ${ }^{1}$; Regina Maria Papais Alvarenga ${ }^{1}$; Helcio Alvarenga Filho ${ }^{1,2}$

${ }^{1}$ Unirio, Hospital Federal Da Lagoa; ${ }^{2}$ UNESA

Introduction: Idiopathic acute transverse myelitis (IATM) is a neurological syndrome resulting from inflammatory involvement of the spinal cord characterized by different degrees of motor, sensory and/ or autonomic dysfunction, with variable remission, monophasic or recurrent course. According to diagnostic criteria IATM can be classified as Isolated clinical syndrome, NMO spectrum syndrome, MOGAb syndrome, or monophasic or idiopathic recurrent IATMs. In Jaoan the spinal form of Asian MS is recognized, where recurrent myelitis occurs, with no dissemination to the central nervous system.

Objectives: To describe a recurrent IATM case report in Brazilian patient with the spinal form of Asian multiple sclerosis (MS).

Results and Conclusions: Case report: Female patient, 36 years old, from Rio de Janeiro/Brazil, married, nurse, with no previous comorbidities, presented paresis and visual disturbance in March/2009, with 31 , evolving tetra paresthesis and autonomic dysfunction in 1 week. Magnetic resonance imaging (MRI) of cervical spine confirmed damage and spine fluid was normal. Underwent pulse therapy with partial response. After 20 days occurs the second attack and the MRI shows a new contrast-enhancing lesion. Three months later, more one attack, when the doctors have chosen to start specific treatment. However, in the period from June/2009 until December/2011, there were more than five new attacks, motivating the exchange of medication for Rituximab. No clinical improvement was evidenced, being replaced in April/2012 for Rebif 44, in use until now, by the evident clinical improvement and low frequency of attacks. Complementary exams did not provide further clarification, and antibodies against AQP4 and anti MOG were negative. In conclusion, we present an infrequent case of MS in a non-Asian population, and indicate the importance of reclassification cases previously idiopathic, mainly after new criteria, because inserted in any form of disease, just allows to initiate a specific treatment.

Funding: No funding or sponsorship was received for this research.

Keywords: Multiple sclerosis 


\section{EPIO10}

CLARITY: An Analysis of Severity and Frequency of Relapses in Patients with Relapsing-Remitting Multiple Sclerosis (RRMS) Treated with Cladribine Tablets 3.5 mg/kg (CT3.5) or Placebo (PBO) [2]

Sven Schippling ${ }^{1}$; Maria Pia Sormani ${ }^{2}$; Nicola De Stefano ${ }^{3}$; Gavin Giovannoni ${ }^{4}$; Andrew Galazka ${ }^{5}$; Birgit Keller ${ }^{6}$; Nektaria Alexandri ${ }^{6}$

${ }^{1}$ University Hospital Zürich, Rämistrasse; ${ }^{2}$ Department of Health Sciences, University of Genoa and Ospedale Policlinico San Martino IRCCS; ${ }^{3}$ Department of Neurological and Behavioural Sciences, University of Siena; ${ }^{4}$ Queen Mary University of London, Blizard Institute, Barts and The London School of Medicine and Dentistry; ${ }^{5}$ Merck, Aubonne, Switzerland, a division of Merck KGaA; ${ }^{6}$ Merck KGaA

Introduction: Treatment with Cladribine Tablets $10 \mathrm{mg}$ [cumulative dose $3.5 \mathrm{mg} / \mathrm{kg}$ (CT3.5)] showed efficacy vs. PBO over 2 years in patients with RRMS (CLARITY).

Objectives: CT3.5 effect on relapse rate and severity (using hospitalisation and steroid use as proxy indicators), and effect of adjusting for covariates was evaluated post hoc.

Methodology: Qualifying relapse was defined by Kurtzke Functional Score status and specified clinical parameters. Qualifying relapse relative risk (RR) was estimated for CT3.5 $(N=433)$ and PBO $(N=437)$ treated patients at Week-24, 48 and 96 by Poisson regression with treatment and various alternating covariates (gender, age, age at time of diagnosis, disease duration, pre-treatment) as main effects and by adding treatment by covariate interaction effects. All relapses were also analysed; analyses were post hoc and exploratory.

Results and Conclusions: Qualifying relapse RR for CT3.5 vs. PBO was lower at Weeks-24, 48 (both $p<0.001)$ and Week-96 $(\mathrm{RR}=0.42$ [95\% confidence interval (CI) $0.34,0.53]$; $p<0.0001)$. All relapse RR (Week-96; CT3.5 vs. PBO) was 0.43 [95\% CI 0.37, $0.51] ; p<0.0001$. Annualised relapse rates for PBO and CT3.5 were 0.35 and 0.15 (qualifying), and 0.63 and 0.27 (all relapses), respectively. CT3.5 vs. PBO reduced risk of qualifying relapses requiring hospitalisation at all timepoints (Week-96 RR $=0.41[95 \%$ CI $0.29,0.57] ; p<0.0001)$ and to steroid treatment (Week-96 RR = 0.41 [95\% CI 0.32, 0.53]; $p<0.0001$ ). Risk reduction of all relapses for hospitalisation and steroid use were 63\% and 62\%, respectively (Week96). Age at time of diagnosis $(p=0.0011)$ and prior use of disease modifying drugs $(p=0.0002)$ significantly affected qualifying relapse rate by Week-96 when added separately to the model. Gender had a marginal effect ( $p=0.0783)$; disease duration had no effect $(p=0.8770)$. No covariate influenced qualifying relapse RR for CT3.5 vs. PBO (Week-96).

Qualifying relapse (and all relapse) RR was consistently and significantly lower with CT3.5 vs. PBO for every timepoint, including relapses requiring hospitalisation or steroid treatment. After adjusting for covariates, treatment benefit of CT3.5 vs. PBO was not diminished by any model adjustments.

Funding: This study was sponsored by EMD Serono Inc, a business of Merck KGaA, Darmstadt, Germany (in the USA), and Merck Serono SA, Geneva, an affiliate of Merck KGaA Darmstadt, Germany (ROW). Keywords: Cladribine tablets; Efficacy; Relapse

\section{EPIO11}

Efficacy of Cladribine Tablets in Patients with Highly Active Relapsing-Remitting Multiple Sclerosis: Analysis of Pooled Double-Blind Data From the CLARITY and ONWARD Studies [3]

Gavin Giovannoni ${ }^{1}$; Xavier Montalban ${ }^{2,3}$; Kottil Rammohan ${ }^{4}$; Stuart Cook $^{5}$; Giancarlo Comi ${ }^{6}$; Peter Rieckmann ${ }^{7}$; Per Soelberg Sørensen ${ }^{8}$; Patrick Vermersch ${ }^{9}$; Fernando Dangond $^{10}$; John King ${ }^{11}$; Doris Damian ${ }^{10}$

${ }^{1}$ Queen Mary University of London, Blizard Institute, Barts and The London School of Medicine and Dentistry; ${ }^{2}$ Division of Neurology, St Michael's Hospital, University of Toronto; ${ }^{3}$ Department of Neurology-Neuroimmunology, Centre d'Esclerosi Múltiple de Catalunya (Cemcat), Hospital Universitario Vall d'Hebron; ${ }^{4}$ University of Miami School of Medicine, Department of Neurology, MS Research Center; ${ }^{5}$ Rutgers, The State University of New Jersey, New Jersey Medical School; ${ }^{6}$ Department of Neurology and Institute of Experimental Neurology, Università Vita-Salute San Raffaele, Ospedale San Raffaele; ${ }^{7}$ Department of Neurology, Medical Park Loipl and University of Erlangen; ${ }^{8}$ Danish MS Center, Department of Neurology, University of Copenhagen; ${ }^{9}$ Université de Lille, CHU Lille, LIRIC-INSERM U995, FHU Imminent; ${ }^{10}$ EMD Serono, Inc.; ${ }^{11}$ Department of Neurology, Royal Melbourne Hospital

Introduction: Patients with relapsing MS (RMS) who show an increased rate of relapse or disability worsening can be described as showing high disease activity (HDA). Treatment with Cladribine Tablets $10 \mathrm{mg}$ [cumulative dose $3.5 \mathrm{mg} / \mathrm{kg}$ (CT3.5)] in the CLARITY study, and with CT3.5 added to interferon- $\beta$ in the ONWARD study demonstrated efficacy vs. placebo (PBO) across a range of patients with active MS. Combining double-blind data from these studies allows assessment of the efficacy of treatment with CT3.5. Objectives: The objective of this post hoc analysis is to compare the effects of CT3.5 vs. PBO on the proportion of patients achieving no evidence of disease activity (NEDA) status in HDA subgroups identified 
using two sets of criteria in a pooled cohort of patients from CLARITY and ONWARD.

Methodology: Patients from CLARITY and ONWARD were retrospectively stratified using 2 sets of HDA criteria based on relapse history, prior treatment, and MRI characteristics: high relapse activity (HRA; $N=314$ ) and HRA plus disease-activity-on-treatment (HRA + DAT; $N=459$ ). Patients treated with CT3.5 or PBO who fulfilled these criteria and achieved NEDA were compared over 2-years using odds ratios (ORs) and 95\% CIs.

Results and Conclusions: In the HRA subgroup, 76.5\% of CT3.5-treated patients were relapse-free, $86.7 \%$ were $\mathrm{T} 1 \mathrm{Gd}+$ lesion-free vs. $50.7 \%$ and $35.8 \%$, respectively, for PBO. In the HRA + DAT subgroup, $77.6 \%$ were relapse-free and $88.8 \%$ were $\mathrm{T} 1 \mathrm{Gd}+$ lesion-free with CT3.5 vs. $53.9 \%$ and $40.8 \%$, respectively for PBO. In the HRA and HRA + DAT subgroups, $42.7 \%$ and $41.3 \%$, respectively, of CT3.5treated patients were disease activity free compared with 9.7\%, (OR 6.94, 95\% CI 3.67; 13.12, $p<0.0001$ ) and $13.7 \%$ (OR 4.28, 95\% CI 2.62; 6.99, $p<0.0001$ ) respectively, of $\mathrm{PBO}$ recipients. In the overall CLARITY + ONWARD population (including nonHDA patients), the composite NEDA score also favoured CT3.5 over PBO (OR 3.95, 95\% CI 2.90; 5.37, $p<0.0001)$. Compared with $\mathrm{PBO}$, treatment with CT3.5 was associated with increased odds of achieving NEDA in HDA patients.

Funding This study was sponsored by EMD Serono Inc, a business of Merck KGaA, Darmstadt, Germany (in the USA), and Merck Serono SA, Geneva, an affiliate of Merck KGaA Darmstadt, Germany (ROW). Keywords: Cladribine tablets; Efficacy; No evidence of disease activity

\section{EPIIO12}

A Longitudinal Study of Cognitive Impairment in Patients with Multiple Sclerosis and its Relationship with Optical Coherence Tomography Parameters

Anna Gil-S ${ }^{1}$; Marc Canudes ${ }^{1}$; Beatriz Vázquez ${ }^{2}$; Hugo Gonzalo $^{2}$; Lara Nogueras ${ }^{3,4}$; Cristina González-Mingot ${ }^{2}$; José Vicente Hervás ${ }^{2}$; Joan Valls ${ }^{1}$; Maria José Solana ${ }^{2}$; Silvia Peralta $^{5}$; Alicia Traveset ${ }^{2}$; Luis Brieva ${ }^{2}$

${ }^{1}$ Institut de Recerca Biomèdica de Lleida; ${ }^{2}$ Hospital Universitari Arnau de Vilanova de Lleida; ${ }^{3}$ Universitat de Lleida; ${ }^{4}$ Departamento de medicina experimental; ${ }^{5}$ Fundació Esclerosi Múltiple de Lleida

Introduction: The cognitive impairment (CI) has been linked to lesions and atrophy in multiple sclerosis (MS). Optical coherence tomography (OCT) is considered an atrophy and progression marker.
Objectives: Our hypothesis is that OCT parameters could be a biomarker for cognitive impairment in MS. Methodology: 206 patients were enrolled and assessed at baseline and after 1 year. To all patients neuropsychological assessment and OCT were performed. The evaluation included the Brief Repeatable Battery of Neuropsychological Test (BRBN) and 4 additional executive function tests. Patients were classified in two groups: CI and preserved. The average thickness of the retinal nerve fiber layer (aRNFL) the quadrants, the volume of the macula cube and the ganglion cell layer (GCL) were analyzed. Results and Conclusions: At baseline, a statistical significance was found in the temporal quadrant of the RNFL which was lower in the group of cognitively impaired patients $(p<0.041)$. Regarding the assessment 1 year later, a statistical significance reduction of the GCL was found in the patients with CI versus those considered preserved $(p<0.041$ and $p<0.049)$. When the changes in CI were analyzed with changes in the OCT parameters, no statistical significance differences were observed. Conclusions: The CI is related to some of the OCT parameters: the temporal quadrant at baseline and the GCL 1 year later. When we analyzed if the change in cognition (from normal to CI) was related to a decrease in OCT parameters we did not observe statistical significance which could indicate that these parameters evolve in a different way.

Funding: No funding or sponsorship was received for this research.

Keywords: Optical coherence tomography; Cognition

\section{EPII013}

Motor Imagery (Home-Based) Effect in Functional Mobility and Fatigue in Patients with Multiple Sclerosis: A Case Series Protocol

Raquel Carvalho ${ }^{1}$; Bruna Silva ${ }^{2}$

${ }^{1}$ Department of Physical Therapy, CESPU, Institute of Research and Advanced Training in Health Sciences and Technologies; ${ }^{2}$ Superior Health School of Vale do Sousa, CESPU, IPSN

Introduction: Patients with multiple sclerosis manifest several signs and symptoms such as numbness, loss of vision, lack of coordination and gait impairment interfering with their functional mobility, and could be associated with fatigue. Motor imagery, i.e. internal rehearsal/simulation of an action without any evident physical movement, has been used to improve gait in neurological conditions, however, 
little research has been carried out in patients with multiple sclerosis.

Objectives: This study aims to explore the effect of a home-based motor imagery intervention, in the fatigue and functional mobility in patients with multiple sclerosis.

Methodology: A single-blind pilot study with a pre-post intervention design. Patients execute the motor imagery home-based during $15 \mathrm{~min}$, three-times-aweek for 4 weeks. Functional mobility and fatigue are assessed pre and post intervention, using the Timed Up and Go and the Modified Fatigue Impact Scale (MFIS), respectively. Assessors are blind to group moment (pre or post).

Results and Conclusions: The idea of enhancing functional mobility and decrease fatigue through the use of motor imagery rehabilitation techniques is important with potential implications for clinical practice. The techniques were developed as a pilot trial for designing larger clinical trials in the future.

Funding No funding or sponsorship was received for this research.

Keywords Mental practice; Lower limb function; Physiotherapy; Gait; Timed up and go

\section{EPIIO14}

Relationship of the Professional Status with Cognitive and Motor Deficits in Patients with Multiple Sclerosis: Exploratory Study

Ana Silva ${ }^{1}$; Daniela Ventura ${ }^{1}$; Telma Miranda ${ }^{2}$; Maria José Sá $^{2,3}$; Cláudia Sousa ${ }^{2}$

${ }^{1}$ Faculdade de Psicologia e de Ciências da Educação da Universidade do Porto; ${ }^{2} \mathrm{MS}$ Clinic, Department of Neurology, Centro Hospitalar Universitário de São João Porto, Alameda Prof. Hernâni Monteiro, Porto, Portugal; ${ }^{3}$ Faculty of Health Sciences, University Fernando Pessoa, Porto, Portugal

Introduction: Motor impairment and cognitive dysfunction in patients with multiple sclerosis (MS) have been associated with their employment status.

Objectives: This study aims to determine in a hospitalbased cohort of MS patients if there is any relationship between the professional status and cognitive and motor functions.

Methodology: The sample is composed by 30 MS patients ( 24 females and 6 males) attended at our MS Clinic from March 2018 to October 2018. The mean age was 39.93 years $(\mathrm{DP}=11.39$; variation $31-$ 61 years) and most patients were employed (57\%). The most prevalent type of MS was the relapsing-remitting (87\%); median EDSS and disease duration were 1.5 (variation $0-6$ ) and 8.3 years (variation
1-28), respectively. Cognition was assessed by the Brief International Cognitive Assessment for Multiple Sclerosis (BICAMS) and Brief Repeatable Battery of Neuropsychological Tests (BRBN-T), functional ability and motor skills with Multiple Sclerosis Functional Composite (MSFC). A Chi square test of independence was performed to examine the distribution of the cognitive impairment by employment status.

Results and Conclusions: Using the criteria of cognitive impairment defined by "two or more abnormal tests", it was found that $43 \%$ of MS patients were impaired, of whom eight inactive and five active, although the difference was not statistically significant. However, when analyzed the performances by each cognitive test with four $t$ tests for independent groups we verified that active patients compared to inactive had significant better results in all screening tests of BICAMS [BVMT $(p=0.011)$; CVLT $(p=0.027)$; SDMT $(p<0.001)]$, and in all BRBN-T tests [RLP $(p$ $<0.001) ; \quad$ CRLP $\quad(p=0.029) ; \quad$ PASAT $\quad(p=0.005)]$ except $10 / 36(p=0.217)$. In MSFC tests, active patients still performed better than the inactive ones [25 foots $(p=0.003) ; 9$ hole-pegs total $(p=0.010)$ ]. This exploratory study raise the hypothesis that professionally active patients have better cognitive performance in memory and speed of information processing trials as well as in motor skills tests. Our limitation is the small sample.

Funding: No funding or sponsorship was received for this research.

Keywords: Employment status; Cognitive impairment; BICAMS; MSFC

\section{EPIIO15}

Cognition, Fatigue and Mood Disorders of Portuguese Patients with Neuromyelitis Optica Spectrum Disorders: The Cohort of Centro Hospitalar São João

Ana Luisa Rocha ${ }^{1,2}$; Daniela Ferro ${ }^{1,2}$; Cláudia Sousa ${ }^{1}$; Teresa Mendonça $^{1}$; Pedro Abreu ${ }^{1,2}$; Joana Guimarães ${ }^{1,2}$; Maria José Sá $a^{1,3}$

${ }^{1}$ Department of Neurology, São João University Hospital Center, Porto, Portugal; ${ }^{2}$ Department of Clinical Neurosciences and Mental Health, Faculty of Medicine, University of Porto, Portugal; ${ }^{3}$ Faculty of Health Sciences, University Fernando Pessoa, Porto, Portugal

Introduction: Neuromyelitis Optica Spectrum Disorders (NMOSD) is a group of rare relapsing inflammatory demyelinating conditions. Cognitive dysfunction, fatigue and mood disorders are well documented in patients in multiple sclerosis (MS), but less characterized in NMOSD. 
Objectives: To characterize cognition, fatigue and mood disorders in patients with NMOSD in our center.

Methodology: Application of Montreal Cognitive Assessment test (MoCA), Brief International Cognitive Assessment for MS (BICAMS), tests of verbal fluency (categorical and phonemic), Hospital Anxiety And Depression Scale (HADS) and Modified Fatigue Impact Scale (MFIS) in our hospital cohort.

Results and Conclusions: Results: We included 11 from 18 NMOSD patients with current follow-up in our center; 9 female, mean age at interview 52 years old (range 23-71). Four patients had positive anti-AQP4 antibodies; 9 had at least one episode of optic neuritis (NO) and 5 had episodes of transverse myelitis (TM) (4 with both NO and TM). Mean result of MoCA test $(n=11)$ was 21 points (SD 7), mean result of CVLT $(n=11)$ was 48.7 words (SD 10.5). Mean number of words in verbal fluency was 23.4 (SD 16.4) in categorical type and 14.6 (SD 5.8) in phonemic type. Mean result of BVMT-R $(n=9)$ was 19.4 (SD 9.0), and mean result of SDMT $(n=8)$ was 43 (SD 15.6). HADS $(n=9)$ showed borderline values for anxiety (mean 9.4, SD 4.8) and for depression (mean 8.7, SD 5.1). Mean total MFIS scale $(n=6)$ result was 43 points (SD 11.7).

Discussion: In our cohort, NMOSD patients showed important cognitive impairment and levels of fatigue, not as much depression and anxiety as expected comparing with studies performed in MS patients. The small number of patients tested is a limitation of our study. In addition, the existence of visual and motor sequels in some patients prevented the completion of all the tests, suggesting that it might be necessary to adapt the tests to this population.

Funding: No funding or sponsorship was received for this research.

Keywords: NMO; NMOSD; Cognition; Fatigue; Mood disorders

\section{EPIIO16}

Lymphopenia Rates in CLARITY/CLARITY Extension are Unrelated to Disease Activity at Baseline [4]

Stuart Cook $^{1}$; Gavin Giovannoni ${ }^{2}$; Patrick Vermersch ${ }^{3}$; Per Soelberg Sørensen ${ }^{4}$; Birgit Cook ${ }^{5}$; Dominic Jack ${ }^{5}$

${ }^{1}$ Rutgers, The State University of New Jersey; ${ }^{2}$ Queen Mary University of London, Blizard Institute, Barts and The London School of Medicine and Dentistry; ${ }^{3}$ Université de Lille, CHU Lille, LIRIC-INSERM U995, FHU Imminent; ${ }^{4}$ Danish MS Center, Department of Neurology, University of Copenhagen; ${ }^{5}$ Merck KGaA

Introduction: In CLARITY, patients with high disease activity (HDA) relapsing multiple sclerosis had clinical and magnetic resonance imaging responses to Cladribine Tablets $10 \mathrm{mg}$ [cumulative dose $3.5 \mathrm{mg} / \mathrm{kg}$ (CT3.5)] that were generally better than/comparable to the overall CLARITY population.

Objectives: Post-hoc analyses to determine lymphopenia rate in patients with HDA in the CLARITY/ CLARITY Extension CT3.5 cohort.

Methodology: Patients were retrospectively analysed using two HDA criteria sets: (1) high relapse activity (HRA): $\geq 2$ relapses in year before study entry, with/ without disease modifying drug (DMD) treatment. (2) HRA plus disease-activity-on-treatment (DAT): $\geq 1$ relapse $\mathrm{AND} \geq 1 \mathrm{~T} 1$ gadolinium-enhancing or $\geq 9 \mathrm{~T} 2$ lesions in year before study entry during treatment with other DMDs. Patients exposed to CT3.5 in Years$1 / 2$ only were the CT3.5 group; the CT7.0 patient group received further courses in Years 3/4. The Adverse Event of Special Interest (AESI) lymphopenia was determined by absolute lymphocyte count (ALC) and lymphopenia from CLARITY/CLARITY Extension.

Results and Conclusions: In CT3.5-Year-2, AESIs/100 patient-years were similar in HRA and HRA + DAT subgroups [11.50 $(N=196) ; 13.09(N=219)]$, and in corresponding non-HDA groups [14.08 $(N=489)$; $13.46(N=466)]$. In CT7.0-Year-4 rates were approximately double to CT3.5-Year-2. Median ALC nadir $\left(\times 10^{9} / \mathrm{L}\right)$ for CT3.5-Year-2 HRA and HRA + DAT were 0.71 and 0.70 ; median nadirs were 0.65 in corresponding non-HDA subgroups. A similar pattern was seen in CT7.0-Year-4, but with lower nadirs than CT3.5-Year-2. Time to ALC nadir differed slightly between HDA subgroups. Incidence of Grade-3 lymphopenia for CT3.5-Year-2 was similar between HDA and non-HDA subgroups, and for CT7.0-Year-4 was lower in HDA than non-HDA subgroups. Grade-3 lymphopenia incidence was lower in CT3.5-Year-2 than CT7.0-Year-4. Grade-4 lymphopenia incidence was low in all subgroups. No relevant differences existed between HDA and non-HDA groups regarding incidence of AESI lymphopenia, ALC nadir, and time to ALC nadir. 
Funding: This study was sponsored by EMD Serono Inc, a business of Merck KGaA, Darmstadt, Germany (in the USA), and Merck Serono SA, Geneva, an affiliate of Merck KGaA Darmstadt, Germany (ROW). Keywords: Cladribine tablets; Lymphopenia; High disease activity

\section{EPIIO17}

Infectious Risk Mitigation in Patients with Multiple Sclerosis Under Disease-Modifying Treatment: The Experience of a Portuguese Tertiary Care Hospital

Daniela Ferro ${ }^{2}$; Beatriz Prista-Leão ${ }^{1}$; Andreia Costa ${ }^{2}$; André Silva-Pinto ${ }^{1}$; Cândida Abreu ${ }^{1}$; Pedro Abreu ${ }^{2}$; Joana Guimarães ${ }^{2}$; Jorge Reis ${ }^{2}$; Teresa Mendonça ${ }^{2}$; António Sarmento ${ }^{1}$; Maria José Sá ${ }^{2}$

${ }^{1}$ Serviço de Doenças Infeciosas do Centro Hospitalar Universitário de São João; ${ }^{2}$ Serviço de Neurologia do Centro Hospitalar Universitário de São João

Introduction: Disease-modifying therapies (DMT) for multiple sclerosis (MS), especially non-BRACE (Betaseron, Rebif, Avonex, Copaxone, Extavia), raise concerns about infectious risk. Our work analyses the result of a systematic infectious diseases (ID) consultation in a cohort of MS patients before or during treatment with these drugs.

Objectives: To understand the importance of ID consultation on patients taking non-BRACE therapies, regarding infectious risk, vaccination strategies and surveillance needs.

Methodology: We collected demographic and clinical data from clinical records of MS patients followed in the MS Clinic and ID consultation (2010-2017).

Results and Conclusions: 149 patients (107 females) planned to start a non-BRACE DMT (natalizumab, fingolimod, dimethyl fumarate (DMF), teriflunomide or rituximab): 19 naïve, 95 previously on BRACE and 35 already under non-BRACE DMT. Median age was 36 years and disease duration was 7.5 years. A diagnosis of latent tuberculosis (LTB) was elicited in 40 patients and $88 \%$ were treated. We found a positive varicella-zoster virus (VZV) IgG or a history of varicella in 133 patients; 80 were immune against hepatitis $B$ virus and 74 to hepatitis A virus. Six patients were vaccinated for VZV; anti-HBV, anti-HAV and anti-pneumococcal vaccines were recommended for those without immunity or previous vaccination. In follow-up, a low $\mathrm{CD}^{+}$count prompted Pneumocystis jirovecii chemoprophylaxis in two patients; recurrent genital herpes motivated chemoprophylaxis in 1 patient. Hospital admission was needed in a patient with chickenpox, a patient with pyelonephritis and in a case of cervicitis; all of them recovered. Other non-severe infections reported were: urinary tract infections (17), skin/soft tissue infections (15), respiratory infections (12) and gastroenteritis (3).

In this cohort, we point the high number of LTB diagnosis and the need for its treatment before starting DMT. This study highlights the importance of individualized management as well as the assessment and mitigation of the infectious risk before and even during these therapies.

Funding: No funding or sponsorship was received for this research.

Keywords: Disease-modifying treatment; Infectious risk; Multiple sclerosis

\section{EPII018}

Peripheral Lymphocyte Count Association with Clinical and Imaging Outcomes in MS Patients Treated with Fingolimod

Manuel Salavisa ${ }^{1}$; Filipa Serrazina ${ }^{1}$; Filipa Ladeira ${ }^{1}$; Ana Sofia Correia $^{1,2}$

${ }^{1}$ Serviço de Neurologia, Hospital Egas Moniz, Centro Hospitalar Lisboa Ocidental; ${ }^{2}$ NOVA Medical School, Faculdade de Ciências Médicas da Universidade Nova de Lisboa

Introduction: Fingolimod prevents lymphocyte egress from lymphoid tissue, thereby reducing CNS autoaggressive lymphocyte infiltration.

Objectives: We aimed to study whether the degree of lymphocyte reduction was associated with fingolimod efficacy, measuring clinical and imaging disease outcomes.

Methodology: Retrospective data analysis of consecutive MS patients currently under fingolimod in our institution, with complete blood count available at baseline and at 3/6 months, minimum period of 1 year follow-up and available MRI data at 1 year follow-up.

Clinical data concerning relapse occurrence, time to relapse, EDSS confirmed progression at 6 months and time to progression was collected from patients' files. Presence of enhancing lesions and T2 lesion load increase at 1 year follow-up MRI was recorded, as well as lymphocyte count at baseline and at 3/6 months. Patients were separated into two groups according to nadir lymphocyte value above/below 500 .

We analyzed the association between lymphocyte value and clinical/imaging variables using Chi square test. We estimated risk of relapse between groups and risk of disability progression using Cox univariate/multivariate regression and Kaplan-Meier survival analysis with log-rank test.

Results and Conclusions: A total of 43 patients were reviewed, 13 excluded due to incomplete data. Twenty-one were female (70\%), mean age of 40.3 years $( \pm 8.7)$ mean EDSS at baseline of 2.8 
$( \pm 1.5)$ and mean duration of disease at baseline of 10 years $( \pm 5.5)$, with no significant differences found between groups. A nadir lymphocyte count below 500 was not associated with the presence of enhancing lesions $(p=0.32)$ or T2 lesion load increase $(p=0.23)$.

Nadir lymphocyte count below 500 was not significantly associated with risk of first relapse (HR 1.10; $95 \%$ CI $0.34-3.60 ; p=0.875)$ or risk of EDSS confirmed progression (HR 0.934; 95\% CI 0.30-2.89; $p=0.905)$.

In our cohort, a higher degree of lymphocyte reduction was not independently associated with imaging outcomes, risk to relapse or risk to EDSS progression Funding: No funding or sponsorship was received for this research.

Keywords: Fingolimod; Lymphopenia; Clinical outcomes; Imaging outcomes

\section{EPIIO19}

Ocrelizumab in a real-world primary progressive multiple sclerosis portuguese population

João Sequeira $^{1,2}$; Joana Parra ${ }^{1}$; Marisa Brum ${ }^{1}$; Ary Sousa ${ }^{1}$; Ariana Barros ${ }^{1}$; Rui Pedrosa ${ }^{1}$; Carlos Capela ${ }^{1}$; Manuel Manita $^{1}$

${ }^{1}$ Serviço de Neurologia, Centro Hospitalar Universitário de Lisboa Central, E.P.E.; ${ }^{2}$ Valência de Neurologia, Centro Hospitalar Barreiro Montijo, E.P.E.

Introduction: Ocrelizumab, a humanized monoclonal antibody targeting CD20-expressing B cells, was approved in the European Union in January 2018 for the treatment of adult patients with relapsing and primary progressive multiple sclerosis (PPMS). The efficacy and safety of ocrelizumab in PPMS patients were assessed during phase III ORATORIO clinical trial.

Objectives: To evaluate the safety, tolerability and effectiveness of ocrelizumab in a real-world clinical setting.

Methodology: Prospective longitudinal cohort study of patients with PPMS treated with ocrelizumab at our center. Demographic and clinical characteristics, including Expanded Disability Status Score (EDSS), Timed 25-Foot Walk (T25-FW), 9-Hole Peg Test (9HPT), Magnetic Resonance Imaging (MRI) data, prior disease modifying therapies, infusion-related reactions and other adverse effects were examined.

Results and Conclusions: Twenty-one patients were included, $11(52.4 \%)$ males with a mean age of $46.1 \pm 9.1$ years. Mean disease duration from symptom onset was $9.1 \pm 6.9$ years and mean disease duration from diagnosis of PPMS was $5.3 \pm 5.9$ years. Median pre-treatment EDSS was 3.5 (range 1.5-7.5), mean T25-FW was $17.8 \pm 27.7 \mathrm{~s}$ and mean 9-HPT (both hands) was $34.1 \pm 17.5 \mathrm{~s}$. Four out of 20 (20.0\%) patients had gadolinium-enhancing lesions on baseline MRI. Eight (38.1\%) patients were treatment-naïve prior to ocrelizumab initiation. Mean follow-up time was $3.7 \pm 2.5$ months with 6 (28.6\%) patients under treatment for at least 6 months. Infusion-related reactions were experienced by 2 $(9.5 \%)$ patients during one of the first two infusions. Two $(9.5 \%)$ patients experienced infections during follow-up period. In conclusion, our results show that our PPMS patients are similar to the ORATORIO trial population, with a slightly higher time to treatment from symptom onset and diagnosis. Ocrelizumab appears to be safe in our real-world cohort, with a lower frequency of infusion- related reactions than those reported in the literature.

Funding: No funding or sponsorship was received for this research.

Keywords: Ocrelizumab; Primary progressive multiple sclerosis; Real-world

\section{EPIIO20}

The Use of Teriflunomide in Relapsing-Remitting Multiple Sclerosis: Real-World Data from a Tertiary MS Center

Daniela Ferro $^{1}$; Andreia Costa ${ }^{1,2}$; Joana Guimarães ${ }^{1,2}$; Jorge Reis ${ }^{1}$; Teresa Mendonça ${ }^{1}$; Pedro Abreu ${ }^{1,2}$; Maria José Sá ${ }^{1,3}$

${ }^{1}$ MS Clinic, Centro Hospitalar Universitário de São João, Porto; ${ }^{2}$ Faculty of Medicine of Porto University, Porto; ${ }^{3}$ Fernando Pessoa University, Porto

Introduction: Teriflunomide is a once-daily oral medication approved to reduce the annualized relapse rate and the risk of disability progression of relapsing-remitting multiple sclerosis (MS).

Objectives: To characterize the population treated with teriflunomide in a tertiary MS Clinic and the safety and efficacy profiles of this drug.

Methodology: We collected clinical data from patients treated with teriflunomide between December 2015 and December 2018 regarding demographic characteristics, treatment background, efficacy and safety data.

Results and Conclusions: We included 52 patients (33 female). Median age at teriflunomide initiation was 47 years-old (IQR 11; no differences between gender) with a median time from diagnosis to treatment of 11 years (IQR 12). Regarding previous MS treatments, 34 patients had been treated with interferon, 12 with 
glatiramer acetate, 5 with dimethyl fumarate, 3 with natalizumab and 10 patients were treatment naïve. The main reasons for initiating teriflunomide were patient preference $(n=29)$ and adverse events of ongoing medications $(n=16)$. There were no differences in EDSS measures at 3 and 6 months posttreatment comparing to the baseline (median EDSS 1.5 at all time points). Main adverse effects were gastrointestinal complaints $(n=13,25 \%)$, hair weakening $(n=10,19 \%)$ and skin reactions $(n=6$, $12 \%)$. Treatment was stopped in 17 patients (33\%) due to gastrointestinal complaints $(n=4)$, skin/hair effects $(n=4)$, increase of liver enzymes $(n=4)$ or other causes $(n=5)$.

Consistent with other studies, teriflunomide was initiated at a median age of 47 years old, yet without differences in age between gender, and the majority of patients had tried another first line treatment. The proportion of adverse effects leading to drug discontinuation seems to be higher than reported in the literature. We highlight the importance of patient information and the need to accomplish the recommendations regarding clinical and analytical monitoring.

Funding: No funding or sponsorship was received for this research.

Keywords: Teriflunomide; Multiple sclerosis; Efficacy; Safety

\section{EPIIO21}

Multiple Sclerosis and Seronegative

Spondyloarthropathies: What is the Best Approach for Treatment?

Marco Fernandes ${ }^{1}$; Bruna Meira ${ }^{1}$; Manuel Salavisa Teixeira ${ }^{1}$; Filipa Ladeira ${ }^{1}$; Marta Guedes ${ }^{2}$; Ana Sofia Correia ${ }^{1}$

${ }^{1}$ Serviço de Neurologia, Hospital de Egas Moniz, Centro Hospitalar de Lisboa Ocidental; ${ }^{2}$ Serviço de Oftalmologia, Hospital de Egas Moniz, Centro Hospitalar de Lisboa Ocidental

Introduction: Demyelinating lesions of the CNS can occur in the setting of systemic autoimmune diseases, such as seronegative spondyloarthropathies (SS), mostly after treatment with TNF antagonists. Coexistence of SS and multiple sclerosis (MS) can pose a diagnostic and therapeutic challenge.

Results and Conclusions: Case 1: A 48-year-old man with a clinical picture of bilateral uveitis and articular pain was diagnosed with ankylosing spondylitis (AS). He started treatment with sulfasalazine and prednisolone. At the age of 54, the patient was seen at the uveitis clinic and ophthalmologic evaluation identified atrophy of the right optic disc. At that time, he reported an episode of painless subacute visual loss in the right eye with incomplete recovery around the age of 42. The patient was then referred for the Neurology clinic. Neurological examination revealed a right relative afferent pupillary defect and mild pyramidal signs. Neuroaxis MRI showed periventricular, infratentorial and spinal cord demyelinating lesions; and CSF analysis with the presence of type 2 and type 4 OCB. He was diagnosed with MS and started treatment with Glatiramer Acetate. During 1 year of treatment, he did not have any relapses of MS, and remained stable regarding AS.

Case 2: A 33-year-old woman with MS since the age of 19 , under treatment with interferon-beta for 11 years, was diagnosed with psoriasis. After 2 years, she presented with synovitis of the metatarsal phalanges and dactylitis, meeting the criteria for psoriatic arthritis. She was started on prednisolone $5 \mathrm{mg} /$ day and sulfasalazine, with articular pain control and no clinical relapses of MS during 21 months of follow-up.

Conclusion: Due to different pathogenesis and immunology pathways of MS and SS, treatment strategy is debatable. There is no evidence-based recommendation for treatment in these patients. IFN-b should be used with caution in patients with MS and SS taking in account IFN-b participation in Th17-mediated diseases.

Funding: No funding or sponsorship was received for this research.

Keywords: Multiple sclerosis; Seronegative spondyloarthropathies

\section{EPIIO22}

Application of the Revised 2005, 2010 And 2017 McDonald Criteria for Multiple Sclerosis in a Portuguese Cohort of Patients with Clinically Isolated Syndrome

Carolina Salgueirinho ${ }^{1}$; Joana Guimarães ${ }^{2,3}$; Teresa Mendonça $^{2}$; Jorge Reis ${ }^{2}$; Maria José Sá ${ }^{2,4}$; Pedro Abreu ${ }^{2,3}$

${ }^{1}$ Faculty of Medicine, Porto University, Portugal; ${ }^{2}$ Department of Neurology, Centro Hospitalar São João; ${ }^{3}$ Department of Clinical Neurosciences and Mental Health, Faculty of Medicine University of Porto; ${ }^{4}$ Faculty of Health Sciences, Hospital Fernando Pessoa

Introduction: The early diagnosis of multiple sclerosis (MS) in the clinical practice is sometimes challenging. Although the McDonald MS diagnosis criteria are, in Portugal, widely applied, data on the sensitivity and specificity of the different criteria in the Portuguese population with Clinically Isolated Syndrome (CIS) is still unavailable. 
Objectives: To evaluate and compare the sensitivity, specificity, positive predictive value and negative predictive value of the 2005, 2010 and 2017 McDonald criteria in a cohort of Portuguese patients with CIS and to evaluate their impact in the early diagnosis of this disease.

Methodology: Retrospective study of CIS patients were followed at the MS clinic of Centro Hospitalar Universitário de São João from January 1, 2007 to December 31, 2017.

Results and Conclusions: 35 CIS patients were included, $23(66 \%)$ were female. Median age at onset and median follow up duration were 27 years (24.00-33.50) and 9.62 months (69.75-113.14), respectively. Twenty-nine patients had a monofocal onset (10 hemispheric syndrome, 7 optic neuritis, 6 brainstem/cerebellar syndrome and 6 spinal cord syndrome). During follow up 25 patients with CIS (71\%) developed Clinically Definitive MS, median time to conversion of 8.67 months, and 34 (97\%) started a disease-modifying drug. At baseline, McDonald 2005 criteria showed a sensitivity of $8 \%$ and a specificity of $100 \%$; McDonald 2010 criteria had a sensitivity of $32 \%$ and a specificity of $50 \%$; $2017 \mathrm{McD}$ onald criteria showed a sensitivity of $64 \%$ and a specificity of $20 \%$.

Our data suggest that the most recent revision, published in 2017, can lead to an earlier MS diagnosis in a higher number of patients with CIS compared to the previous criteria revisions. Nevertheless, the specificity seems to suffer a significant reduction.

Funding: No funding or sponsorship was received for this research.

Keywords: Clinically isolated syndrome; Multiple sclerosis; Diagnostic criteria; McDonald criteria.

\section{EPIIIO23}

Mitochondrial Redox Status is Impaired in the Peripheral Lymphocytes of Multiple Sclerosis Patients

Lara Nogueras ${ }^{1}$; Hugo Gonzalo ${ }^{2}$; Anna Gil ${ }^{3}$; Jose Vicente Hervás ${ }^{4}$; Petya Valcheva ${ }^{3}$; Cristina González ${ }^{4}$; Meri Martín ${ }^{3}$; Meri Canudes ${ }^{3}$; Jose Vicente Peralta ${ }^{4}$; Maria Jose Solana ${ }^{4}$; Reinald Pamplona ${ }^{1}$; Manel Portero ${ }^{1}$; Jordi Boada ${ }^{1}$; Jose Serrano $^{1}$; Luis Brieva ${ }^{4}$

${ }^{1}$ Universitat de Lleida; ${ }^{2}$ Instituto de Salud de Castilla y Leon; ${ }^{3}$ IRB Lleida; ${ }^{4}$ Hospital Universitario Arnau de Vilanova de Lleida

Introduction: Multiple sclerosis (MS) is a demyelinating inflammatory disease of the central nervous system (CNS) with an etiopathology still under discovery. It is known that immune system is directly involved in MS physiopathology and since oxidative stress (OS) is essential in immune function, several reports indicate CNS samples from MS patients and models exhibit OS-derived changes. However, whether this is present in peripheral immune cells and the potential mechanisms behind MS-linked OS are unknown. The aim of this study is to find out whether OS can be detected in peripheral lymphocytes of MS patients in comparison to controls, and what could be the basis of this OS.

Objectives: We performed a cross-sectional, observational pilot study ( $n=58,34 \mathrm{MS}$ and 24 control (CTL) individuals). We used flow cytometry to identify lymphocyte populations and superoxide quantitation (median of fluorescence intensity, MFI); immunoblotting for mitochondrial complexes; commercial kits for lactate detection and ABTS technique for plasma antioxidant capacity ( $\mu \mathrm{M}$ equivalents of Trolox). Comparisons between study groups were adjusted by potential confounders.

Methodology: Lymphocytes from MS patients exhibit a significant increase in the superoxide anion production in comparison to controls: adjusted mean difference $(95 \% \mathrm{CI})=330.9(17.4 ; 644.4)$ MFI. We also observed significant decreased contents of representative peptides from mitochondrial respiratory complexes I, III and V and decreased plasma antioxidant capacity, suggesting antioxidant exhaustion in these patients: adjusted mean difference $(95 \% \mathrm{CI})=-$ 128.8 (- 216.6; - 41) $\mu \mathrm{M}$ equivalents of Trolox. In line with compromised mitochondrial function, MS patients also exhibited increased plasma lactate concentration: adjusted mean difference $(95 \% \mathrm{CI})=4.07$ (0.98; 7.16) $\mathrm{mg} / \mathrm{dL}$.

Results and Conclusions: Our findings suggest that part of the widely reported OS at CNS level, could be related with an impaired mitochondrial redox status in the peripheral lymphocytes of MS patients. Therefore, lymphocyte infiltration could drag this characteristic increasing locally OS in CNS.

Funding: No funding or sponsorship was received for this research.

Keywords: Mitochondria; Oxidative stress; Immune system; Multiple sclerosis 


\section{EPIIIO24}

\section{Magnetic Resonance Imaging in Multiple Sclerosis (MAGNIMS) Score Predicts Long-Term Clinical Disease Activity (CDA)-Free Status and Disability Progression in Subcutaneous Interferon Beta-1a (scIFNß-1a)-Treated Patients [5]}

\begin{abstract}
Maria Pia Sormani ${ }^{1}$; Mark S. Freedman ${ }^{2}$; Julie Aldridge ${ }^{3}$; Kurt Marhardt $^{4}$; Nicola De Stefano ${ }^{5}$

${ }^{1}$ Department of Health Sciences, University of Genoa and Ospedale Policlinico San Martino IRCCS; ${ }^{2}$ University of Ottawa and the Ottawa Hospital Research Institute; ${ }^{3}$ EMD Serono Research \& Development Institute, Inc.; ${ }^{4}$ Merck GmbH; ${ }^{5}$ Department of Neurological and Behavioural Sciences, University of Siena
\end{abstract}

Introduction: scIFN $\beta$-1a reduces relapse rates and delays disability progression in patients with multiple sclerosis (MS).

Objectives: This post hoc analysis of scIFN $\beta$-1a-treated patients from the PRISMS study examines the association of Year (Y) 1 MAGNIMS score on time to CDA event and disease progression.

Methodology: In PRISMS-2, relapsing-remitting MS patients were randomized to scIFN $\beta-1 \mathrm{a} 22$ or $44 \mu \mathrm{g}$, or placebo, three times weekly for 2 years. Placebo patients were randomized to scIFN $\beta-1$ a $22 / 44 \mu \mathrm{g}$ at Y3. Patients were followed to Y15 post-randomization $(22 \mu \mathrm{g} n=95 ; 44 \mu \mathrm{g} n=95$; placebo $n=100)$. We classified scIFN $\beta-1$ a patients by Y1 MAGNIMS score: 0 (0-2 new T2 lesions +0 relapses), 1 (0-2 new T2 lesions +1 relapse or $>3$ new T2 lesions +0 relapses) or $2(0-2$ new $\mathrm{T} 2$ lesions $+\geq 2$ relapses or $\geq 3$ new T2 lesions $+\geq 1$ relapse). CDA-free was defined as no relapses or disability progression (increase of 1 point from baseline in Expanded Disability Status Scale (EDSS) score, or 1.5 points in patients with EDSS $0)$. Median times [95\% confidence interval (CI)] to first CDA event and EDSS progression from Y1, and respective hazard ratios [HR (95\% CIs)] versus MAGNIMS score of 0 , are presented.

Results and Conclusions: At Y1, 129, 108 and 130 scIFN $\beta$ - 1 a patients had MAGNIMS score of 0,1 and 2 , respectively. Median time to CDA event was longer in patients with Y1 MAGNIMS score of 0 (2.6 [2.1-3.5] years) vs. 1 (1.7 [1.5-2.0] years; $\mathrm{HR}=1.7$ [1.3-2.3]) and 2 (1.3 [1.2-1.4] years; HR = 2.4 [1.9-3.2]). Median time to EDSS progression was longer in patients with Y1 MAGNIMS score of 0 (7.5 [6.9-13.5] years) vs. 1 (4.0 [3.5-7.5] years; $\mathrm{HR}=1.5[1.1-2.1])$ and $2(2.5$ [1.9-3.5] years; $\mathrm{HR}=2.1$ [1.6-2.9]). In PRISMS, MAGNIMS score at Y1 predicted risk of CDA event or disability progression in scIFN $\beta$-1a-treated patients. Funding: This study was sponsored by EMD Serono Inc, a business of Merck KGaA, Darmstadt, Germany (in the USA), and Merck Serono SA, Geneva, an affiliate of Merck KGaA Darmstadt, Germany (ROW).
Keywords: MAGNIMS; Scoring system; Interferon beta; Treatment response; Disability progression; Clinical disease activity.

\section{EPIIIO25}

Pregnancy in Multiple Sclerosis: An Unsuspected Double-Edged Sword?

Maria João Pinto ${ }^{1,2}$; Mafalda Seabra ${ }^{1,2}$; Maria José Sá ${ }^{1,3}$; Joana Guimarães ${ }^{1,2}$

${ }^{1}$ Department of Neurology, Centro Hospitalar Universitário de São João, Porto, Portugal; ${ }^{2}$ Department of Clinical Neurosciences and Mental Health, Faculty of Medicine, University of Porto, Porto, Portugal; ${ }^{3}$ Faculty of Health Sciences, University of Fernando Pessoa, Porto, Portugal

Introduction: Several studies revealed lower multiple sclerosis relapse rates during pregnancy. Diseasemodifying therapies are generally avoided during this period.

Results and Conclusions: In 2010 a 19-year-old woman with no relevant past history presented to emergency department with left hemiparesis, dysphagia, mild dysphonia and left limbs dysmetria. CSF analysis revealed mild pleocytosis, normal glycose and protein levels, unmatched oligoclonal bands and negative infectious study. Brain MRI confirmed multiple subcortical, periventricular and infratentorial white matter lesions. Nine months later new hemispheric T2 white matter lesions were apparent on brain MRI, some with gadolinium enhancement. Accordingly, the diagnosis of relapsing-remitting multiple sclerosis was admitted, but she refused treatment initiation. After a spinal cord relapse, she started treatment with interferon $\beta$-1a; however, 5 years later, treatment was interrupted due to twin pregnancy. She experienced no relapses during gestation (EDSS 1) and refused to resume treatment (including intravenous immunoglobulin) during lactation (12 months), despite a new relapse during puerperium. She is currently pregnant again and clinically stable (EDSS 1 ). The opposite clinical scenario occurred in a 27-yearold woman admitted after several episodes of right hemisensory loss and ipsilateral limbs motor deficit. CSF analysis was unremarkable apart from unmatched oligoclonal bands. T2-weighted brain and spinal cord MRI showed widespread periventricular, subcortical and infratentorial white matter hyperintense lesions. Assuming the diagnosis of relapsing-remitting multiple sclerosis, treatment with interferon $\beta-1 \mathrm{a}$ was started. Due to ongoing disease activity, it was switched to natalizumab 3 years later, achieving disease stability (EDSS 1, stable lesion burden) during the next year. Therefore it was decided to stop immunomodulatory therapy in order to get 
pregnant. During pregnancy she experienced several relapses (EDSS 4). She resumed treatment with natalizumab shortly after giving birth, as she continued experiencing relapses.

Conclusion: We presented two cases of active multiple sclerosis with two different courses during pregnancy, confirming its heterogeneity and unpredictability.

Funding: No funding or sponsorship was received for this research.

Keywords: Multiple sclerosis; Pregnancy; Diseasemodifying therapy

\section{EPIII026}

Longitudinally Extensive Transverse Myelitis in Lupus: Is it Another Autoimmune Disorder?

Ricardo Soares-Dos-Reis ${ }^{1,2,3,4}$; Bruno Fernandes ${ }^{5}$; Miguel Bernardes $^{5}$; Maria José Sá ${ }^{1,6}$; Joana Guimarães ${ }^{1,2,3,7}$

${ }^{1}$ Neurology Department, Centro Hospitalar Universitário São João, Porto, Portugal; ${ }^{2}$ Department of Clinical Neurosciences and Mental Health, Faculdade de Medicina da Universidade do Porto, Portugal; ${ }^{3}$ Department of Biomedicine, Faculdade de Medicina da Universidade do Porto, Portugal; ${ }^{4} \mathrm{i} 3 \mathrm{~S}$, Institute for Research and Innovation in Health, University of Porto, Portugal; ${ }^{5}$ Rheumatology Department, Centro Hospitalar Universitário São João, Porto, Portugal; ${ }^{6}$ Faculty of Health Sciences, University Fernando Pessoa, Porto, Portugal; ${ }^{7}$ Center for Drug Discovery and Innovative Medicines (MedInUP), University of Porto, Portugal

Introduction: Neuropsychiatric lupus occurs in up to $40 \%$ of systemic lupus erythematosus (SLE) patients. However, central nervous system involvement in SLE patients may occur as a result of other autoimmune conditions, such as anti-NMDA receptor autoimmune encephalitis or neuromyelitis optica spectrum disorders (NMOSD).

Methodology: A 41-year-old male came to our attention complaining of shock-like pain in his limbs, numbness in his legs and mild urinary incontinence. His prior medical history included: Buerger's disease, treated with sympathectomy; SLE, diagnosed 6 years prior in the context of migratory arthralgias, fatigue, oral ulcers, positive ANA, anti-dsDNA and Coombs test, low complement and hypergammaglobulinemia; and recently diagnosed interstitial lung disease. He was treated with hydroxychloroquine $400 \mathrm{mg}$ /day and folate. His neurological examination showed preserved higher functions, no involvement of cranial nerves, mild paresis of his left arm and both legs, pinprick and vibratory hypesthesia in his lower limbs and left C5-C8 dermatomes, with globally brisk reflexes. Neuraxis magnetic resonance imaging (MRI) showed no brain lesions (including double inversion recovery sequences) and extensive myelitis showing disperse, mainly peripheral, hyperintense T2 lesions with gadolinium enhancement (C2, C7, D1-D3, D6-D8 and D11). Cerebrospinal fluid analysis showed mild inflammation ( 8 cells $/ \mathrm{mm}^{3}$ ) and negative oligoclonal bands. Intravenous methylprednisolone was started with partial symptom resolution and patient was maintained on a steroid taper and pregabalin. Azathioprine was introduced later when positivity for anti-AQP4 antibodies was known. At his last appointment, MRI showed resolution of spinal cord T2 hyperintensities. However, the patient maintained paroxysmal tonic spasms, ameliorated by a second course of steroids.

Results and Conclusions: This patient illustrates the overlap of NMOSD with SLE, in line with recent cohort data, where up to half of SLE-related myelitis were anti-AQP4 positive. Thus, this case adds further evidence to the usefulness of screening for anti-AQP4 antibodies in SLE patients with myelitis.

Funding: No funding or sponsorship was received for this research.

Keywords: Systemic lupus erythematous; Neuromyelitis optica

\section{EPIIIO27}

Anti-MOG Antibody Associated Central Nervous System Diseases: Expanding the Spectrum

Cláudia Melo ${ }^{2}$; Ricardo Soares Dos Reis ${ }^{1}$; Jacinta Fonseca ${ }^{2}$; Luis Augusto ${ }^{3}$; Joana Guimarães ${ }^{1}$; Maria José Sá ${ }^{1}$; Mafalda Sampaio $^{2}$; Dilio Alves ${ }^{1}$; Raquel Sousa ${ }^{2}$

${ }^{1}$ Neurology Department, Centro Hospitalar Universitário São João, Porto, Portugal; ${ }^{2}$ Child Neurology Unit, Pediatric Department, Centro Hospitalar Universitário São João, Porto, Portugal; ${ }^{3}$ Neuroradiology Department, Centro Hospitalar Universitário São João, Porto, Portugal

Introduction: Antibodies against myelin oligodendrocyte glycoprotein (MOG) have been described in neuromyelitis optica spectrum disorders, multiple sclerosis, acute disseminated encephalomyelitis and, less frequently, in viral encephalitis.

Methodology: Case 1: A 6-year-old boy was admitted due to diplopia. Two weeks earlier he was diagnosed with acute sinusitis and treated with amoxicillinclavulanate. On admission, he presented diplopia, right sixth nerve palsy, and bilateral papilledema. Blood workup showed leukocytosis and a CRP of $83 \mathrm{mg} / \mathrm{L}$. CSF: 17 leukocytes $/ \mathrm{mm}^{3}$, normal glucose and protein. Brain CT showed bilateral white matter hypointensities. Presumptive treatment with aciclovir and ceftriaxone was started. Brain MRI (day 2) revealed hyperintensity lesions (T2 and FLAIR) on fronto-temporo-parieto-occipital regions, thalamus and brainstem, some were contrast-enhancing. 
Methylprednisolone pulse therapy was started with success. A later MR angiography raised the hypothesis of post-vasculitis lesions. MOG antibodies were positive (antiAQP4-neg and negative oligoclonal bands). Case 2: A 16-year-old female, was admitted due to headache, nausea and loss of vision. Initial workup, including blood workup and brain CT were normal. Some days later she started vomiting and presented ataxia and a pyramidal syndrome. Brain MRI showed large T2/FLAIR cortico-subcortical and pontine hyperintense and contrast-enhanced lesions, and an hyperintense optic chiasm lesion. Spinal MRI revealed several enhancing lesions. VEPs: bilateral decreased amplitude and increased latency. CSF: 20 leukocytes $/ \mathrm{mm}^{3}$, glucose and protein were normal. MOG antibodies and oligoclonal bands were positive (antiAQP4-neg). Methylprednisolone therapy (5 days, $1 \mathrm{~g} /$ day), oral prednisolone and rituximab were prescribed with gradual recovery. During steroid tapering, motor deficits worsened and intravenous immunoglobulin was started with improvement.

Results and Conclusions: We present two illustrative cases of the wide spectrum of anti-MOG antibody disease. A positive response to immunosuppression is remarkable. However, there are reports in the literature of a high incidence of relapses during steroid taper, as in case 2 . Specific pediatric treatment protocols are necessary for better therapeutic management. Funding: No funding or sponsorship was received for this research.

Keywords: Anti-MOG; Encephalomyelitis.

\section{EPIIIO28}

Anti-MOG Antibody-Positive Isolated Longitudinally Extensive Transverse Myelitis

Adilson Cláudio Marcolino ${ }^{1}$; Vera Cabreira ${ }^{1,3}$; Teresa Mendonça $^{1}$; Cátia Figueiredo ${ }^{2}$; Goreti Nadais ${ }^{1}$

${ }^{1}$ Neurology Department, São João University Hospital Centre, Porto; ${ }^{2}$ Medicine Internal Department, Tondela, Viseu, Hospital Centre, Viseu; ${ }^{3}$ Neurociences Department, Faculty of Medicine, University of Porto

Introduction: The finding of the serum diagnostic biomarkers like aquaporin- 4 autoantibody targeting astrocytes and myelin oligodendrocyte glycoprotein autoantibody (MOG-IgG) targeting oligodendrocytes has changed the paradigm of the classification of what was previously known as idiopathic transverse myelitis (TM). TM is frequently an early manifestation of MOG-IgG-related disease and it may occur in isolation, as a component of acute disseminated encephalomyelitis (ADEM) or concurrently with optic neuritis. The report of MOG-IgG-related TM cases is valuable as information for its specific features is still very limited.

Case Report: 50-year-old man, with a concurrent history of diabetes and arterial hypertension presented a clinical presentation with a two-week course of gait imbalance, numbness in abdomen and lower members and urinary retention which had been preceded by influenza-like prodromal symptoms. On neurological examination, there was a prominent flaccid tetraparesis and a upper thoracic level by T4. Cerebrospinal fluid showed mononuclear pleocytosis (440 cells $/ \mu \mathrm{L})$ and elevated protein concentration. Other laboratory tests, including extensive viral serologies were otherwise normal/negative. MRI spine demonstrated a longitudinally extensive T2 hyperintensity running in the superior-inferior direction from the conus to bulbomedullary transition on sagittal images. Brain MRI disclosed a few periventricular lesions inconsistent with ADEM. None of the lesions demonstrated gadolinium enhancement. He was administered high- dose intravenous methylprednisolone (1 g/day, 8-day course) with significant improvement and functional recovery. After initiation, a MOG-IgG seropositivity was identified and 5 months after he did not experience any relapses.

Results and Conclusions: Clinical features such as male sex, a prodromal infectious syndrome, severe motor deficits despite good clinical recovery, neurogenic bladder, longitudinally extensive sagittal T2 lesions, absence of gadolinium enhancement and conus involvement are clues to suspect on a MOG-IgG myelitis. Recognition of this syndrome may have important treatment and prognostic value and help define an adequate individualized follow-up plan.

Funding: No funding or sponsorship was received for this research.

Keywords: Anti-MOG antibody-positive 


\section{EPIIIO29}

Magnetic Resonance Imaging Findings at Optic Neuritis of Recurrent Optic-Spinal Phenotype, Multiple Sclerosis in a Cohort of Brazilian Patients from Rio De Janeiro [6]

Anna Christiany Brandão ${ }^{1}$; Rafaella Eliria Abbott Ericksson ${ }^{1}$; Ana Carolina Ribeiro Araujo E. Araujo ${ }^{1}$; Nadja Emidio De Araujo $^{1}$; Nathalie Stephanie Meneguette ${ }^{1}$; Silvia Bastos De Oliveira $^{1}$; Claudia Cristina Vasconcelos ${ }^{1}$; Cleonice Bento ${ }^{1}$; Marcos Papais Alvarenga ${ }^{1}$; Ana Claudia Bahia De Andrade ${ }^{1}$; Claudio Henrique Lopes ${ }^{1}$; Paulo Roberto Valle Bahia ${ }^{1}$; Marcell Pourbaix Morrisson ${ }^{1}$; Carla Regina Marchon ${ }^{1}$; Tadeu $\mathrm{Kubo}^{1}$; Patricia Piazza Rafful ${ }^{1}$; Thomas Doring ${ }^{1}$; Lara Alexandre Brandão ${ }^{2,3}$; Sergio Luis Shimdt ${ }^{1}$; Gustavo Tukamoto $^{1}$; Regina Maria Papais Alvarenga ${ }^{1}$

${ }^{1}$ Unirio, Hospital Da Lagoa; ${ }^{2}$ Radiologic Department, Clínica Felippe Mattoso, Fleury Medicina Diagnóstica; ${ }^{3}$ Radiologic Department, Clínica Irm-Ressonância Magnética

Introduction: Optic neuritis may evolve into multiple sclerosis and MRI demonstrates lesions in optic nerves.

Objectives: To study MRI of optic nerves.

Methodology: Patients with recurrent optic neuritis (ON) with a good recovery and transverse myelitis (TM) were followed from 2000 to 2015, 10 years of disease and EDSS $<3$, were classified as "benign" with a recurrent optic spinal phenotype multiple sclerosis (OSMS). A prospective population study was performed on 16 patients OSMS. All patients was negative (AQP4)-IgG and negative (MOG)-IgG. Most patients showed brain and spine lesions met MAGNIMS criteria (2016). For lesions on MRI in sequence, STIR with the optic nerves were divided into three segments: intra-orbital (IO) $(25 \mathrm{~mm}$ : the longest segment and communication between subarachnoid space around the optic nerve that in suprasellar cistern, canalicular (CAN) (9 mm: as through optic canal and prechiasmatic and chiasmal (CHI) $(16 \mathrm{~mm}$ : intracranial segment in suprasellar cistern).

Results and Conclusions: 2 (12.5\%) patients showed normal optic nerves. Lesions on MRI the optic nerves in $9(56.25 \%)$ patients with lesion unilateral, 5 (31.2\%) patients with lesions bilateral, $14(87.5 \%)$ patients with lesion intra-orbital (IO), 7 (43.7\%) showed lesion CAN, 14 (87.5\%) IO and 1(6.2\%) patient showed lesion prechiasmatic. No patient showed contrast enhancement. Patients with recurrent $\mathrm{ON}$ and $\mathrm{TM}$, at least 10 years of disease and low EDSS indicate that optic spinal recurrent phenotype of MS and not a NMO spectrum syndrome. Most patients did not have extensive lesions in optic nerves and visual event good recovery could be classified as a subgroup of MS with a benign course.
Funding: No funding or sponsorship was received for this research.

Keywords: Magnetic resonance imaging; Neuritis; Multiple sclerosis

\section{EPIIIO30}

MRI Findings of Recurrent Optic Spine Phenotype Multiple Sclerosis in a Cohort of Patients from Rio De Janeiro, Brazil: Clinical and Laboratorial Evaluation

Anna Christiany Brandão ${ }^{1}$; Rafaella Eliria Abbott Ericksson ${ }^{1}$; Ana Carolina Ribeiro Araujo E. Araujo ${ }^{1}$; Nadja Emidio De Araujo $^{1}$; Nathalie Stephanie Meneguette ${ }^{1}$; Silvia Bastos De Oliveira ${ }^{1}$; Claudia Cristina Vasconcelos ${ }^{1}$; Cleonice Bento ${ }^{1}$; Marcos Papais Alvarenga ${ }^{1}$; Ana Carolina Bahia De Andrade ${ }^{1}$; Vanderson Nery ${ }^{1}$; Carlos Henrique Lopes Basoni ${ }^{1}$; Romulo Varella De Oliveira ${ }^{1}$; Emerson Leandro Gasparetto ${ }^{1}$; Carolina De Medeiros Rimkus²; Ana Celia Baptista Koifman ${ }^{1}$; Paulo Roberto Valle Bahia ${ }^{1}$; Lara Alexandre Brandão $^{3}$; Patricia Piazza Rafful ${ }^{1}$; Sergio Luis Shimdt ${ }^{1}$; Carla Regina Marchon ${ }^{1}$; Tadeu Kubo ${ }^{1}$; Gustavo Tukamoto ${ }^{1}$; Thomas Doring ${ }^{1}$; Marcell Pourbaix Morrisson ${ }^{1}$; Regina Maria Papais Alvarenga ${ }^{1}$

${ }^{1}$ Unirio, Hospital Da Lagoa; ${ }^{2}$ Universidade De São Paulo, USP; ${ }^{3}$ Departamento De Radiologia Clinica Fellipe Mattoso

Introduction: The NMO spectrum covers different demyelinating condition as NMO (2006) and optic Asian optic spine MS (OS-MS) (1996) that were characterized by recurrent optic neuritis and transverse myelitis, and positivity of the anti-AQP4 antibody. Clinical and laboratorial features are different in NMO spectrum syndrome and multiple sclerosis.

Objectives: Describe MRI findings in OS-MS patients with clinical and laboratorial evaluation.

Methodology: Retrospective and descriptive study. In the cohort of patients with recurrent optic neuritis and transverse myelitis were followed from 2000 to 2016 in Hospital Lagoa and Hospital Gafrée Guinle (Rio de Janeiro, Brazil). The OS-MS patients were selected patients did not meet criteria to the NMO (2006). At least one brain and one spinal cord MRI for each patient was analysed by two neuroradiologists to evaluate images (blind study).

We analysed the following clinical variables and laboratory data, duration of disease and classification of lesions observed by MRI. The MS criteria (2016) and NMO spectrum disorder (NMOSD) (2015) were applied.

Results and Conclusions: In 41 patients, only 27 were included, because no have requisitions the search. In OS-MS patients sample of 27 patients, the majority female and white, mean age at onset 29.8 years. Mean time of disease 16.1 year. Number of events 269 (TM 199, ON 70). First event: ON in 56\%. Only $9.75 \%$ 
developed second progression. OS-MS. Patients with 10 years of disease and EDSS $\leq 3$, were classified as "benign" and the anti-AQP4 was negative. The OS-MS patients $(100 \%)$ showed radiological criteria for MS in all cases and only two patients had extensive spinal cord lesions and all patients presented optic nerve lesions.

Our findings indicate that clinical and laboratorial data of OS-MS were optic spinal recurrent phenotype meet MS criteria and not a NMO spectrum syndrome. The OS-MS patients could be classified as subgroup of MS relapsing-remitting with benign course.

Funding: No funding or sponsorship was received for this research.

Keywords: Magnetic resonance imaging; Recurrent optic spine; Multiple sclerosis

\section{EPIIIO31}

Metabolic Diseases: A Differential Diagnosis of Primary Progressive Multiple Sclerosis

Célia Nogueira ${ }^{5}$; Diogo Ribeiro ${ }^{1}$; Maria José Sá ${ }^{6}$ j Joana Guimarães $^{4}$; Mafalda Seabra ${ }^{4}$; Maria Carmo Macário ${ }^{2}$; Ana Martins Silva ${ }^{3}$; Laura Vilarinho ${ }^{5}$

${ }^{1}$ Unidade de Investigação e Desenvolvimento, Departamento de Genética Humana, Instituto Nacional de Saúde Doutor Ricardo Jorge, Porto; ${ }^{2}$ Centro Hospitalar e Universitário de Coimbra, EPE; ${ }^{3}$ Centro Hospitalar e Universitário do Porto, EPE; ${ }^{4}$ Departamento de Neurociências Clínicas e Saúde Mental, Faculdade de Medicina da Universidade do Porto; ${ }^{5}$ Unidade de Rastreio Neonatal Metabolismo e Genética, Departamento de Genética Humana, Instituto Nacional de Saúde Doutor Ricardo Jorge, Porto; ${ }^{6}$ Faculdade de Ciências da Saúde, Universidade Fernando Pessoa, Porto

Introduction: Multiple sclerosis (MS) is a chronic demyelinating neurological disease primarily affecting young adults, with a prevalence of approximately $0.1 \%$ in the Caucasian population. In recent years some studies have raised concern over the possibility of misdiagnosis in MS, which could be as high as 6\%, particularly among patients with primary progressive MS. Several single gene disorders share clinical and radiologic characteristics with MS, and have the potential to be overlooked in the differential diagnostic evaluation of both adult and pediatric patients. Diagnosis of primary progressive MS has special challenges as there are no relapses and the MRI findings are different from those patients with relapsing onset MS. Clinically primary progressive MS is similar to spastic paraparesis like hereditary spastic paraparesis or other metabolic disorders, such as lysosomal storage disorders, mitochondrial diseases or neurometabolic disorders, presenting with this predominant symptom.

Objectives: The overall aim of our research project is to develop a Next Generation Sequencing strategy to identify metabolic disorders in 104 patients with a presumptive diagnosis of primary progressive MS.

Methodology: Next Generation Sequencing have been performed in a MiSeq Illumina instrument using a custom mitochondrial gene panel with around 250 genes involved in mitochondria and lysosomal metabolism. Libraries have been prepared using SureSelect QXT target enrichment system from Agilent.

Results and Conclusions: This study contributed to identify pathogenic mutations in $7 \%$ of the studied patients in genes associated with hereditary spastic paraparesis and other metabolic disorders.

The uniqueness of this project is to bring NGS technology to the bedside in the management of MS-like conditions, helping clinicians who have patients with diseases for which a diagnosis has been elusive. Recognition of a single-gene disorder as causal for a patient's 'multiple sclerosis-like' phenotype is critically important for effective patient management, and has broad genetic counseling implications for affected families.

Funding: This research was sponsored by MERCK. Keywords: Metabolic diseases; Primary progressive multiple sclerosis

\section{EPIIIO32}

Concentration Analysis of Metallic Nanoparticles in Multiple Sclerosis Patients

Marcela De Oliveira ${ }^{1}$; Marina Piacenti Da Silva ${ }^{1}$; Fernando Coronetti Gomes Da Rocha ${ }^{2}$; Paulo Noronha Lisboa-Filho ${ }^{1}$

${ }^{1}$ Departamento de Física da Faculdade de Ciências, Universidade Estadual Paulista "Júlio de Mesquita Filho" Campus de Bauru, SP, Brazil; ${ }^{2}$ Departamento de Neurologia, Psicologia e Psiquiatria da Faculdade de Medicina de Botucatu, Universidade Estadual Paulista "Júlio de Mesquita Filho" Campus de Botucatu, SP, Brazil

Introduction: Engineered nanoparticles (ENPs) are objects with dimensions about hundreds of nanometers, which dimension is smaller than animal and plant cells. In general, people are exposed to nanometer-sized metals particles, modernly present in cosmetics, pharmaceuticals, food and in the air. Metallic ENPs have the potential to penetrate in human cells and cause neurodegenerative diseases. Multiple sclerosis (MS) is a multifactor neurological disease that could be associated to the exposure to metallic elements; however, the present literature 
still needs to trace possible relation between the present of metallic elements in the blood and this pathology.

Objectives: The aim of the present study was to analyze the concentration of metallic ENPs in blood samples from patients with multiple sclerosis and healthy individuals.

Methodology: Inductively coupled plasm optical emission spectrometry (ICP-OES) was used to determine the concentrations of three metallic elements (copper, iron and zinc) in blood of 30 patients with MS compared with healthy individuals.

Results and Conclusions: We observed reduction in concentrations levels for copper and zinc in multiple sclerosis patients. Zinc results were $0.132 \mathrm{mg} / \mathrm{L}$ for MS patients and $0.213 \mathrm{mg} / \mathrm{L}$ for control group. Our cooper results showed a concentration of $0.007 \mathrm{mg} / \mathrm{L}$ in blood of patients and $0.021 \mathrm{mg} / \mathrm{L}$ for healthy individuals. Blood iron levels were significantly elevated in MS group $(15.3 \mathrm{mg} / \mathrm{L}$ for multiple sclerosis patients and $13.5 \mathrm{mg} / \mathrm{L}$ for healthy individuals). According to our data a possible imbalance of these three metallic elements can be considered as a possible cofactor in the multiple sclerosis.

Funding: This research was sponsored by Fundação de Amparo à Pesquisa do Estado de São Paulo (FAPESPprocess number 2017/20032-5 and process number 2015/22864-2).

Keywords: Metallic nanoparticles; Multiple sclerosis; ICP-OES; Zinc; Copper; Iron

\section{EPIIIO33}

Ethnic-Racial Characteristics in Multiple Sclerosis Patients in the City of Rio De Janeiro

Fabiana Carolino ${ }^{1,2}$; Raphael Guimarães ${ }^{4}$; Alexandre Lucidi $^{5}$; Helcio Avarenga Filho ${ }^{1}$; Regina M Papais Alvarenga ${ }^{3}$

${ }^{1}$ Department of Neurology, Graduate Program in Neurology (PPGNEURO), Universidade Federal do Estado do Rio de Janeiro-UNIRIO, Rio de Janeiro; ${ }^{2}$ Rio de Janeiro; ${ }^{3}$ Department of Neurology, Graduate Program in Neurology (PPGNEURO), Universidade Federal do Estado do Rio de Janeiro-UNIRIO, Rio de Janeiro, Brazil; ${ }^{4}$ Oswaldo Cruz Foundation, Rio de Janeiro, Brazil; ${ }^{5}$ Department of Neurology, Graduate Program in Neurology (PPGNEURO), UNIRIO, Brazil

Introduction: The distribution of multiple sclerosis (MS) worldwide cannot be fully explained by biologic or ambiental factors. Brazil is a tropical South American country with low MS prevalence. By self-declaration, most Brazilian are "black, white, yellow, brown and indigenous" (Instituto Brasileiro de Geografia e Estatística, IBGE), with high miscegenation due to intermarriage between European colonization, African slaves and indigenous.
Objectives: The objective of this study was to describe the race and ancestry in Rio de Janeiro (RJ) MS patients. Methodology: Patients living in RJ, assisted at the MS main center were selected in 2018 for the study. Race, and phenotypic features in Afro Brazilian ("mulato" or black) and white Brazilians were registered. Patients completed a self-declaration questionnaire according to IBGE (black, white, brown, indigenous and yellow) and information about European or Afro Brazilian family data until the third generation were analysed. Results and Conclusions: In the study, $155 \mathrm{MS}$ patients were included, 79 (51\%) classified as Afro Brazilian (brown and black) and 76 (49\%) as White Brazilian. According self-declaration data, 20 were black, 60 brown, 74 white and one indigenous. In Afro Brazilian group, 14/79 (17.7\%) declared as white and in White Brazilian group 16/76 (22.3\%) self-declared as brown or black. Family history were referred in $26.4 \%$ as European ancestry in 41/155 (12/80 self declared brown/black and 29/74 white). Afro descendents were referred in 53\% from the total $82 / 155$ (62/80 as brown or black, $19 / 74$ as white and one as indigenous). The majority MS patients in RJ are Afro descendant. The agreement between the phenotypic evaluation and self- declaration was 80\%. One-quarter reported European parents or grandparents and more than $50 \%$ identified at least one brown or black ancestor up to the third generation.

Funding: No funding or sponsorship was received for this research.

Keywords: Multiple sclerosis; Ancestry; Race; Ethnicity; Brazil

\section{EPIV034}

Immune Thymic Profile of the MOG-Induced Experimental Autoimmune Encephalomyelitis Mouse Model [7]

Sofia P. Das Neves ${ }^{1,2}$; Cláudia Serre-Miranda ${ }^{1,2}$; Claudia Nobrega $^{1,2}$; Susana Roque ${ }^{1,2}$; João J. Cerqueira ${ }^{1,2,3}$; Margarida Correia-Neves ${ }^{1,2}$; Fernanda Marques ${ }^{1,2}$

${ }^{1}$ Life and Health Sciences Research Institute (ICVS), School of Medicine, University of Minho, Campus Gualtar, 4710-057 Braga, Portugal; ${ }^{2}$ ICVS/3B's, PT Government Associate Laboratory, Braga, Guimarães, Portugal; ${ }^{3}$ Clinical Academic Center-Braga, Braga, Portugal

Introduction: Multiple sclerosis (MS) is a chronic, immune-mediated, demyelinating disease that affects the neurons of the central nervous system. Activated $\mathrm{T}$ cells, specific for myelin epitopes, cross the brain barriers, and react against the myelin sheath, leading to demyelination.

Objectives: Since $\mathrm{T}$ cells are generated within the thymus, here we explored, in mice, the alterations 
occurring in this organ throughout the different phases of the disease.

Methodology: We induced experimental autoimmune encephalomyelitis (EAE) in C57BL/6 females and sacrifice them at the onset (day 16) and chronic phases of disease (day 23), along with non-induced controls.

Results and Conclusions: We observed thymic atrophy in EAE mice at the onset that remained until the chronic phase of disease. This atrophy was associated with a preferential loss of the $\mathrm{CD} 4^{+} \mathrm{CD}^{+}$double positive thymocytes, an intermediate population between the more immature $\mathrm{CD} 4^{-} \mathrm{CD} 8^{-}$double negative and the most mature single positive thymocytes. This was accompanied by an increase in the thymic medullary/cortical ratio and by an altered expression levels of genes important for T cell survival. During the chronic phase, the thymi remained atrophic, but reacquired the normal proportion of the main four thymocyte populations and the normal medullary/cortical ratio. Importantly, at the onset phase, and accompanying these thymic alterations, EAE animals presented an increased percentage of demyelinating lesion area in the cerebellum, and an increased expression of interferon gamma (Ifng), interleukin (Il) 12a, and Il17a. This study suggests dynamic thymic alterations occurring in response to EAE, from the induction to the chronic phase, that might help to elucidate the MS pathophysiology.

Funding: This work was supported by Foundation for Science and Technology (FCT) and COMPETE through the project EXPL/NEU-OSD/2196/2013 and by The Clinical Academic Center (2CA-Braga) through the project EXPL/001/2016. The work at ICVS/3B's has been developed under the scope of the project NORTE-01-0145-FEDER-000013, supported by the Northern Portugal Regional Operational Programme (NORTE 2020), under the Portugal 2020 Partnership Agreement, through the European Regional Development Fund (FEDER), and funded by FEDER funds through the Competitiveness Factors Operational Programme (COMPETE), and by National funds, through the Foundation for Science and Technology (FCT), under the scope of the project POCI-01-0145-FEDER-007038. FM is an assistant researcher and recipient of an FCT Investigator grant with the reference IF/00231/2013. CN and SR are recipients of post-doctoral fellowships with the references SFRH/BPD/112001/2015 and SFRH/BPD/ $72710 / 2010$, respectively, from POPH through FSE and MCTES national funds. CS-M. and SdN are recipients of Ph.D. fellowships with the references SFRH/ $\mathrm{BD} / 112494 / 2015$ and $\mathrm{PD} / \mathrm{BD} / 114120 / 2015$, respectively, from MCTES national funds.

Keywords: Autoimmunity; Multiple sclerosis; Experimental autoimmune encephalomyelitis; Thymus

\section{EPIV035}

\section{Uncommon Presentation of Neurosarcoidosis}

Renata Souto Silva ${ }^{1}$; Mariana Branco ${ }^{1}$; Rita Rodrigues ${ }^{1}$; Elsa Meireles $^{2}$; Mafalda Santos ${ }^{2}$; Luis Ruano ${ }^{1,3}$

${ }^{1}$ Neurology Department, Centro Hospitalar de Entre Douro e Vouga, Santa Maria da Feira, Portugal; ${ }^{2}$ Internal Medicine Department, Centro Hospitalar de Entre Douro e Vouga, Santa Maria da Feira, Portugal; ${ }^{3}$ Departamento de Ciências da Saúde Pública e Forenses e Educação Médica, Faculdade de Medicina da Universidade do Porto, Porto, Portugal

Introduction: Neurosarcoidosis is a rare neuro-inflammatory disorder with protean manifestations which presents a diagnostic challenge.

Objectives: We describe an atypical presentation of neurosarcoidosis, with cerebrospinal fluid (CSF) and histopathological findings.

Methodology: A 40-year-old woman with a 5-month history of fatigue, intermittent fever, skin lesions, lymphadenopathies and arthralgias presented with a rapidly progressive course of confusion and altered behaviour. On admission she was restless, disoriented, with disorganized speech, but without focal neurological deficits or meningism. Later she became very agitated requiring sedation, intubation and admission to the intensive care unit (ICU). A presumptive diagnosis of viral encephalitis was made, and the patient was started on intravenous acyclovir. Brain CT and contrast-enhanced MRI were normal. CSF analysis showed lymphocytic predominant pleocytosis, hyperproteinorrachia and one oligoclonal band. FDG-PET revealed increased FDG uptake of axillary, hilar, inguinal and iliac lymph nodes. An inguinal lymph node biopsy showed histopathological findings of non-caseating granuloma without evidence of malignant cells or infection, supporting the diagnosis of sarcoidosis. Mildly increased levels of serum angiotensin-converting enzyme were found. Extensive CSF and serum studies were negative for infectious diseases, particularly for tuberculosis, malignancy and other autoimmune causes.

The patient was started on $300 \mathrm{mg}$ of hydrocortisone for 8 days with a subsequent 2 months oral prednisolone taper with complete recovery. Two months after completing treatment she presented with erythematous lesions on the shin, leg and forearm that were clinically suggestive of erythema nodosum. She initiated treatment with hydroxychloroquine with complete resolution of skin lesions.

Results and Conclusions: We report a case of severe aseptic encephalitis requiring ICU admission as the first manifestation of neurosarcoidosis in a patient without a previous diagnosis. CSF examination revealed a neuro-inflammatory process. FDG-PET identified a target for biopsy, which allowed 
histological confirmation of noncaseating granulomas. Complete work up is often challenging but essential in establishing a diagnosis that leads to optimal treatment.

Funding: No funding or sponsorship was received for this research.

Keywords: Neurosarcoidosis; Aseptic encephalitis

\section{EPIV036}

Neuromyelitis Optica Spectrum Disorders:

A Diagnosis to Consider Beyond the Age of 75

Ana João Costa ${ }^{1}$; Rui Araújo ${ }^{2}$; Paulo Ferreira ${ }^{2}$; Maria José Sá $^{3,4}$; Joana Guimarães ${ }^{2,5}$

${ }^{1}$ Centro de Reabilitação do Norte; ${ }^{2}$ Serviço de Neurologia, Centro Hospitalar Universitário de São João, Porto, Portugal; ${ }^{3}$ Serviço de Neurologia, Centro Hospitalar Universitário de São João, Porto, Portugal; ${ }^{4}$ Faculdade de Ciências da Saúde, Universidade Fernando Pessoa, Porto, Portugal; ${ }^{5}$ Departamento de Neurociências Clínicas e Saúde Mental, Faculdade de Medicina da Universidade do Porto, Portugal

Introduction: Neuromyelitis optica spectrum disorders (NMOSD) are a rare and highly disabling group of autoimmune disorders with a median age of onset of 40 years old. Even though 25\% of NMOSD patients may have a pediatric or a late onset, there are few case reports beyond the age of 75 .

Clinical Case: We report the case of an 87 year old woman, with a past medical history of hypertension and dyslipidemia, who presented with subacute asymmetric paraparesis, a bilateral sensory level below D6 with impaired gait and urinary retention. The first symptoms reported were pain and numbness of the left limb with progression over a week. A spinal cord MRI was performed and showed T2 hyperintense central cord lesion spanning over nine segments (C4-D6) with dorsal gadolinium enhancement. She was treated with high doses of intravenous methylprednisolone and plasma exchange, with some improvement. During the first plasma exchange sessions, the patient developed dyspnea with bronchospasm that were pharmacologically corrected. Serum Aquaporin 4 antibodies (AQP4-Ab) were positive (1: 3200, cell-based immunofluorescence assay) and a diagnosis of AQP4-Ab positive NMOSD was established. Additionally, matching IgG oligoclonal bands (OCB) in both serum and cerebrospinal fluid with a "mirror pattern" were detected. During the hospitalization, the patient received the first session of rituximab, without complications.

Conclusions: Currently, there are few cases of very late onset NMOSD reported. This case illustrates the importance of considering NMOSD as a possible cause of longitudinally extensive myelitis, even in this age group. Despite the inconclusive investigation for an underlying cancer, NMOSD in elderly patients are infrequent findings that should always raise the suspicion of a paraneoplastic aetiology.

Funding: No funding or sponsorship was received for this research.

Keywords: Longitudinally extensive myelitis; Neuromyelitis optica spectrum disorders.

\section{EPIVO37}

Sphincter Dysfunction: Think of MOG

Mafalda Seabra ${ }^{1,2}$; Daniela Ferro ${ }^{1,2}$; Goreti Nadais ${ }^{1}$; Lucinda Sequeira $^{1}$; Maria José Sá ${ }^{1,3}$; Joana Guimarães ${ }^{1,2}$

${ }^{1}$ Department of Neurology, São João University Hospital Center, Porto, Portugal; ${ }^{2}$ Department of Clinical Neurosciences and Mental Health, Faculty of Medicine, University of Porto, Portugal; ${ }^{3}$ Faculty of Health Sciences, University Fernando Pessoa, Porto, Portugal

Introduction: Longitudinally extensive myelitis (LEM) was, for a couple of years, found almost as a synonym of Neuromyelitis Optica/Neuromyelitis optica spectrum disorders, whether AQP4 antibodies were found or not. With the growing knowledge of the MOG spectrum disorders, some features of LEM may suggest this diagnosis.

Objectives: To review two cases of MOG positive myelitis.

Methodology: Case report.

Results and Conclusions: Case 1: a 52-year-old man presented to the emergency department in May 2017 complaining of altered sensation in the chest, legs, and inability to run. On examination, he had a T5 sensory level. Spinal cord MRI showed a D2-D7 lesion. After 7 days of methylprednisolone, the patient got better. He was readmitted 1 month later. At that time the sensory symptoms worsened as well as urinary and sexual dysfunction. He was again treated with methylprednisolone and was started on Rituximab. MOG antibodies were later found to be positive. The last MRI showed marked improvement, showing just a slight hyperintensity, although the patient still complains of sexual dysfunction.

Case 2: A 32 year old woman was admitted to the Neurology ward with a 2 months history of altered sensation in the legs, urge incontinence and progressive paraparesis. Spinal cord MRI showed a gad enhancing conus medullaris lesion. She was treated with methylprednisolone, intravenous immunoglobulin, and Rituximab. MOG-IgG was positive. At last check up the patient was able to walk without assistance, but still had significant urogenital symptoms. The latest spinal cord MRI showed 
improvement of the distal spinal cord hyperintensity and edema. The presence of predominant urinary symptoms, disproportionate to motor and sensory symptoms, in some cases with conus medullaris lesions, has been found in some patients with MOG antibodies and may suggest this diagnosis. During follow up there may be an improvement of imaging findings unaccompanied by symptoms resolution. Funding: No funding or sponsorship was received for this research.

Keywords: MOG; Myelitis

\section{EPIV038}

\section{Recurrent Optic Neuritis}

Renata Souto Silva ${ }^{1}$; Rita Rodrigues ${ }^{1}$; Marta Lopes ${ }^{2}$; Carlos Veira $^{1}$; Eva Brandão ${ }^{1}$

${ }^{1}$ Neurology Department, Centro Hospitalar de Entre Douro e Vouga, Santa Maria da Feira, Portugal; ${ }^{2}$ Neurology Department, Hospital da Senhora da Oliveira, Guimarães, Portugal

Introduction: Optic neuritis $(\mathrm{ON})$ in multiple sclerosis (MS) is caused by demyelination of the optic nerve and typically presents with acute to subacute unilateral vision loss and pain with eye movements. Visual acuity recovers to $20 / 40$ or better in $92 \%$ of patients. Objectives: A 21-year-old woman presented with sudden RE visual loss, abnormal color vision and ocular pain. She was treated with intravenous methylprednisolone with complete recovery. CSF contained five oligoclonal IgG bands without corresponding bands in serum. MRI showed a slight hyperintense right optic nerve on T2 and FLAIR, multiple supratentorial T2 lesions and one contrast-enhancing infratentorial lesion. MS was diagnosed after screening for mimics and she was started on glatiramer acetate. Her past medical history was unremarkable, and her mother has relapsing-remitting MS.

Methodology: Case report. Three months later she developed left leg paresthesia, with spontaneous remission. Two months afterwards she had subacute painless RE visual loss, with a slight improvement after another methylprednisolone. Brain MRI revealed new supratentorial T2 lesions and T1 hypointense lesions. She was switched to natalizumab.

During the following 5 months, the RE visual acuity had gradually worsened to 20/400. This time, MRI revealed new spinal cord lesions comparatively to the first MRI. Due to this atypical ON, Leber hereditary optic neuropathy (LHON) was suspected and a homoplasmic mutation m.11778G $>$ A was found. The patient started treatment with idebenone.
Results and Conclusions: This patient presented with a typical episode of ON which led to MS diagnosis. The severe visual impairment and lack of recovery warned the possibility of LHON as the cause in the second episode. LHON in association with MS (LHON-MS) is suggested to be a distinct entity that resembles relapsing-remitting MS, with the exception of more frequent and severe involvement of the optic nerves. LHON-MS should be considered in MS patients with atypical vision loss for $\mathrm{ON}$.

Funding: No funding or sponsorship was received for this research.

Keywords: Leber hereditary optic neuropathy; Multiple sclerosis

\section{EPIV039}

CADASIL Mimicking Multiple Sclerosis or Two Concomitant Diseases?

Carolina Soares $^{1,2}$; Ana Luísa Rocha ${ }^{1,2}$; José Dias Da Costa ${ }^{3}$; Fernando Silveira ${ }^{1}$; Luís Gonçalves ${ }^{4}$; Maria José Sá ${ }^{1,5}$; Pedro Abreu $^{1,2}$

${ }^{1}$ Neurology Department, Centro Hospitalar Universitário de São João, Porto, Portugal; ${ }^{2}$ Department of Clinical Neuroscience and Mental Health, Faculdade de Medicina da Universidade do Porto, Portugal; ${ }^{3}$ Neuroradiology Department, Centro Hospitalar Universitário de São João, Porto, Portugal; ${ }^{4}$ Ophthalmology Consultation, Hospital da Luz, Guimarães, Portugal; ${ }^{5}$ Fernando Pessoa University, Porto, Portugal

Introduction: Cerebral autosomal dominant arteriopathy with subcortical infarcts and leukoencephalopathy (CADASIL) is an inherited small vessel disease, manifesting as recurrent stroke, migraine with aura, behavioural and cognitive disturbances. In early stages, CADASIL may be misdiagnosed as multiple sclerosis (MS). Although these two distinctive diseases are not known to co-occur, there are cases of CADASIL with an inflammatory-like presentation, including optic neuritis and spinal cord involvement that can perfectly mimic MS.

Objectives: Case Report.

Methodology: A 25-year-old man started an acute right sensorimotor loss with complete remission within a few days. He has maternal family history of depression, dementia and MS. All autoimmune and infectious diseases were excluded, oligoclonal bands, aquaporin 4 antibody and visual evoked potentials (VEP) were negative/normal. Brain MRI depicted hyperintense lesions at splenium of corpus callosal, left subcortical frontal lobe and periventricular temporal lobe, without gadolinium enhancement or diffusion restriction. Three years later, his 53-year-old 
mother performed a brain MRI due to complaints of migraine and cognitive impairment that depicted white matter abnormalities suggestive of CADASIL and a pathogenic Notch3 mutation was identified. Our patient was later confirmed to be a carrier as well. At 32 years-old, he developed left eye pain and visual loss; VEP revealed prolonged latency on left eye. He started corticotherapy with good response. At this point, CSF oligoclonal bands and plasma aquaporin 4 antibody remains negative, but brain and spinal MRI showed new T2-hyperintense lesions on left optic nerve and in multiple cervical and dorsal spinal segments suggestive of an inflammatory pattern, fulfilling criteria of temporal and spatial dissemination for MS.

Results and Conclusions: The pathological and genetic bases of CADASIL and MS are distinct. In rare cases, clinical spectrum of CADASIL encompasses an inflammatory-like presentation. However, in this case, a demyelinating disease cannot be excluded and may lead to a different approach and treatment.

Funding: No funding or sponsorship was received for this research.

Keywords: CADASIL; Inflammatory-like pattern; Multiple sclerosis; Optic neuritis; Spinal cord

\section{EPIV040}

Familial Multiple Sclerosis: Retrospective Study of 11 Families

Vanessa Oliveira $^{1}$; Sara Duarte ${ }^{1}$; Raquel Samões ${ }^{1}$; Ana Paula Sousa $^{2}$; Andreia Bettencourt ${ }^{3,4}$; Bárbara Leal ${ }^{3,4}$; Berta Martins Da Silva ${ }^{3,4}$; Ana Martins Silva ${ }^{1,5}$; Ernestina Santos ${ }^{1,5}$

${ }^{1}$ Neurology Department, Centro Hospitalar Universitário do Porto, Hospital de Santo António (CHUP-HSA); ${ }^{2}$ Neurophysiology Department, Centro Hospitalar Universitário do Porto, Hospital de Santo António (CHUP-HSA); ${ }^{3}$ Unit for Multidisciplinary Research in Biomedicine, ICBAS, University of Porto, Portugal; ${ }^{4}$ Immunogenetics Laboratory, ICBAS, University of Porto, Portugal; ${ }^{5}$ Unit for Multidisciplinary Research in Biomedicine, ICBAS, University of Porto, Portugal

Introduction: Multiple sclerosis (MS) is the most frequent inflammatory demyelinating CNS disease, with most cases occurring sporadically. Some patients have a first, second or third degree relative also affected by MS, being considered as familial MS.

Objectives: Our aim was to characterize our cohort of patients with familial MS.

Methodology: A retrospective analysis of a MS patients cohort, followed in a Portuguese tertiary center, with at least two affected patients in the same family (related in first degree) was performed.
Results and Conclusions: From a cohort of 655 MS patients, we found a total of 11 families with 2 affected relatives (total of 22 patients). Five families were constituted by a pair of siblings, and the other 6 by a parent-child pair. Patients had an average age of 44 years, $60 \%$ being man. Eighteen patients had relapsing-remitting MS, 3 had secondary progressive MS, 1 had primary progressive MS and 1 had a clinically isolated syndrome. Mean age at onset was 31 -years-old. In the 6 families represented by 2 generations, mean age of onset was 42 years-old in the parents, and 25 years-old in the successive generation. HLA analysis was performed in 18 patients (7 families), with both affected members of each family having at least one haplotype in common. The most common genetic findings were HLA-DRB ${ }^{*} 13$ allele (7 patients) and HLA-DRB ${ }^{*} 15$ allele (6 patients). Only 1 patient had another auto-immune disease. Mean EDSS at the last observation was 3, with an average follow-up of 11 years.

In our cohort, second generation patients seemed to have a lower age at the onset of the disease. Some authors have previously described this finding.

Funding: No funding or sponsorship was received for this research.

Keywords: Multiple sclerosis; Familial multiple sclerosis

\section{EPIV041}

\section{NOTCH3 Mutations in Previously Diagnosed MS Patients}

Mafalda Seabra ${ }^{3,4}$; Daniela Ferro ${ }^{3,4}$; Célia Nogueira ${ }^{1,2}$; Laura Vilarinho $^{1,2}$; Joana Guimarães ${ }^{3,4}$; Maria José Sá ${ }^{4,5}$

${ }^{1}$ Unidade de Investigação e Desenvolvimento, Departamento de Genética Humana, Instituto Nacional de Saúde Doutor Ricardo Jorge, Porto; ${ }^{2}$ Unidade de Rastreio Neonatal Metabolismo e Genética, Departamento de Genética Humana, Instituto Nacional de Saúde Doutor Ricardo Jorge, Porto; ${ }^{3}$ Departamento de neurociências clínicas e saúde mental, Faculdade de Medicina da Universidade do Porto, Porto, Portugal; ${ }^{4}$ Serviço de Neurologia, Centro Hospitalar e Universitário de São João, Porto, Portugal; ${ }^{5}$ Faculdade de ciências da sáude, Universidade Fernando Pessoa, Porto, Portugal

Introduction: Multiple sclerosis (MS) diagnosis is one of exclusion. Several entities can mimic it. One example is Cerebral Autosomal Dominant Arteriopathy and Subcortical Ischemic Leukoencephalopathy (CADASIL), featuring significant white matter abnormalities and neurologic dysfunction.

Objectives: To review three cases of NOTCH3 mutations in patients with an MS diagnosis.

Methodology: Next Generation Sequencing using a custom mitochondrial gene panel with around 250 
genes involved in mitochondria and lysosomal metabolism. Libraries were prepared using SureSelect QXT target enrichment system from Agilent.

Results and Conclusions: Case 1: Female, 62 years-old, followed since 2003 for unspecific neurologic symptoms and white matter lesions, interpreted as MS. In 2011 a diagnostic revision was made and a family history of early dementia was disclosed. Genetic testing and skin biopsy confirmed a CADASIL diagnosis.

Case 2: Female, 58 years-old, diagnosed with MS at 28 when she presented with vertigo. Oligoclonal bands were negative and brain MRI showed periventricular and subcortical hyperintensities. She was treated with interferon beta 1a and remained stable. The diagnosis was reviewed in 2011. There was a history of headaches and genetic testing described a novel NOTCH3 mutation in exons 3 and 32 .

Case 3: Male, 58 years-old, had an episode of leftsided hemiparesis and paresthesia in 1994, interpreted as an MS relapse. Since 2005 he started showing depressive symptoms and cognitive decline. Spinal cord MRI was normal and brain MRI showed extensive white matter lesions. Genetic testing performed in 2009 showed no mutations in the hotspot exons of NOTCH3. In 2017 he had an ischemic stroke. Genetic testing was extended to all exons of NOTCH3, and a novel mutation in exon 6 was found. The authors aim to shed light on 3 cases of prolonged MS misdiagnosis, later redefined as CADASIL. This distinction is many times challenging and a continuous effort to rethink a given diagnosis is recommended.

Funding: No funding or sponsorship was received for this research.

Keywords: CADASIL; Multiple sclerosis

\section{EPIV042}

Natural History of Multiple Sclerosis in a Long Lasting Cohort From a Tertiary MS Centre: Baseline Results

Maria José Sá1,2; Ana Macedo ${ }^{3,4}$; Lucinda Sequeira ${ }^{1}$; Daniela Ferro $^{1,5}$; Mafalda Seabra ${ }^{1,5}$; Ana Luisa Rocha ${ }^{1,5}$; Adilson Marcolino ${ }^{1}$; Cláudia Sousa ${ }^{1}$; Pedro Afonso ${ }^{4}$; Jorge Reis ${ }^{1}$; Teresa Mendonça ${ }^{1}$; Pedro Abreu ${ }^{1,5}$; Joana Guimarães ${ }^{1,5}$; Edite Rio ${ }^{1}$

${ }^{1}$ Department of Neurology, Centro Hospitalar Universitário São João, Porto, Portugal; ${ }^{2}$ Faculty of Health Sciences, University Fernando Pessoa, Porto, Portugal; ${ }^{3}$ Department of Biomedical Sciences and Medicine, Algarve University, Portugal; ${ }^{4}$ Keypoint, Consultoria Científica, Portugal; ${ }^{5}$ Department of Clinical Neurosciences and Mental Health, Faculty of Medicine, University of Porto, Portugal
Introduction: The knowledge of the natural history of multiple sclerosis (MS) is essential to define prognosis and optimize healthcare. In the era of disease-modifying drugs (DMD) the MS history relies upon analysis of standardized long lasting registries.

Objectives: To describe the MS patients followed in a tertiary MS Centre and compare baseline characteristics according to the first appointment date throughout 10-years spans (1987-1996; 1997-2006; 2007-2016).

Methodology: Retrospective study of the MS patients followed in Centro Hospitalar São João: first appointment date, gender, age at diagnosis, disease classification, Expanded Disability Status Scale (EDSS) scores.

The comparisons between three decennials were performed using Chi-square test, ANOVA and Kruskal-Wallis test.

Results and Conclusions: 661 adult patients were included. $70.2 \%$ female; mean age 47.9 years (12.9 y), mean age at diagnosis $34.8 \mathrm{y}$ (sd $11.2 \mathrm{y}$ ), mean disease duration $12.9 \mathrm{y}$ (sd $8.2 \mathrm{y}$ ). The median relapses number was 3 (1-34). Baseline median EDSS was 1.5 (0-8.5). At the first evaluation 93\% were classified as CIS and 6\% as PPMS. At the second evaluation 86\% were classified as RRMS, 3\% as PPMS and 1\% as SPMS. No significant differences were found regarding gender, disease classification and age at diagnosis in patients 87-96 $(n=48), 97-06(n=284)$ and 07-16 $(n=285)$.

Baseline EDSS was significantly higher in 87-96 cohort when compared with the others and higher in the 97-06 cohort than in 07-16 (median 3.0, 1.5 and 1.0). Median time to the first MS treatment showed a significant difference $(85,7$ and 1 months, respectively).

In our MS cohort the baseline patients' characteristics were quite similar along a timespan of 30 years, with the exception of a higher disability found in earlier years, which may be due to the introduction of DMD in 1996. The comprehensive analysis of MS history, DMD effect and other demographic data will further proceed.

Funding: This work was supported by a grant by Merck.

Keywords: Multiple sclerosis; Registry; Natural history 


\section{EPIV043}

Dimethyl Fumarate (DMF) Persistence in Regular Clinical Practice: Strategy to Minimize Adverse Effects

Ana Rodríguez Regal ${ }^{1,2}$; María Del Campo Amigo Jorrín ${ }^{3}$; Luis Anibarro García ${ }^{4}$; Alfredo Puy Nuñez ${ }^{3}$

${ }^{1}$ Department of Neurology; ${ }^{2}$ EOXI PONTEVEDRA-O SALNÉS, Spain; ${ }^{3}$ Department of Neurology, EOXI PONTEVEDRA-O SALNÉS, Spain; ${ }^{4}$ Department of Internal Medicine, EOXI PONTEVEDRA-O SALNÉS, Spain

Introduction: Dimethylfumarate (DMF) has demonstrated efficacy in the treatment of relapsing- remitting Multiple Sclerosis (RRMS) patients. DMF reduces relapses, disability progression and MRI activity for more than 9 years. The principal cause of treatment discontinuation is due to adverse events (flushing, nausea, vomiting and diarrhea).

Objectives: To assess the efficacy and adverse effects of DMF in our patients. To check our strategy effectiveness to minimize adverse effects (AE) and their impact in therapy discontinuation.

Methodology: Retrospective observational study. Participants: all patients with relapsing- remitting multiple sclerosis (RRMS) treated with DMF in our unit. Basal demographic, clinical and radiological data were collected at $6,12,18$ and 24 months, as well as AE. All patients received a written prescription of escalated-dosage and the therapeutic measures to follow in the event of an AE.

Results and Conclusions: We analyzed 51 patients (80\% women) with an average of 35.6 (18-65) years at the beginning of the therapy. From this group, 23 were naïve, 15 therapy failures, 12 intolerant, 1 pregnant. The number of episodes in the previous year was 1 (0-3) and baseline median EDSS 1 (0-4). 68.8\% of the patients had follow-up for over 12 months. One patient's follow-up was missing. At the end of the study, 94\% of the patients showed no episode and 98\% showed equal or improved EDSS. Twenty-four patients had MRI, $80 \%$ out of them remained unchanged or improved; $64 \%$ of patients presented $\mathrm{AE}$, none of them severe. Only 3 patients quit the therapy ( 1 for own decision, 1 for therapy failure, 1 for wishing pregnancy).

Conclusion: In our series, DMF showed high efficacy. $\mathrm{AE}$ were present in $64 \%$ of the patients but, using strategies to minimize them, the patients developed real expectations that made our series free from discontinuation due to AE.

Funding: No funding or sponsorship was received for this research

Keywords: Dimethyl fumarate; RRMS; Efficacy; Tolerability

\section{EPIV044}

Magnetic Resonance Imaging Findings at AntiMyelin Oligodendrocyte Glycoprotein (MOG) Antibodies in a Brazilian Woman with One Severe Bilateral Episode of Optic Neuritis

Anna Christiany Brandao Nascimento ${ }^{1}$; Rafaella Elira Abbott Ericksson $^{1}$; Ana Carolina Ribeiro Araujo E. Araujo ${ }^{1}$; Nathalie Stephanie Meneguette ${ }^{1}$; Nadja Emidio De Araujo ${ }^{1}$; Silvia Bastos De Oliveira ${ }^{1}$; Claudia Cristina Vasconcelos ${ }^{1}$; Cleonice Bento $^{1}$; Marcos Papais Alvarenga ${ }^{1}$; Ana Claudia Bahia De Andrade ${ }^{1}$; Carlos Henrique Lopes Basoni ${ }^{1}$; Vanderson Neri ${ }^{1}$; Romulo Varella De Oliveira ${ }^{1}$; Emerson Leandro Gasparetto ${ }^{1}$; Carolina De Medeiros Rimkus²; Ana Celia Baptista

Koifman ${ }^{1}$; Paulo Roberto Valle Bahia ${ }^{1}$; Lara Alexandre Brandão $^{3}$; Patricia Piazza Rafful ${ }^{1}$; Sergio Luis Shimdt ${ }^{1}$; Carla Regina Marchon ${ }^{1}$; Tadeu Kubo ${ }^{1}$; Gustavo Tukamoto ${ }^{1}$;

Thomas Doring ${ }^{1}$; Marcell Pourbaix Morrisson ${ }^{1}$; Regina Maria Papais Alvarenga ${ }^{1}$

${ }^{1}$ Unirio, Hospital Federal Da Lagoa; ${ }^{2}$ Universidade De São Paulo, USP; ${ }^{3}$ Clinica Radiologica Fellipe Mattoso

Introduction: Myelin oligodendrocyte glycoprotein (MOG) localizes on the outermost surface of the myelin sheath and oligodendrocytes in the central nervous system (CNS).

Objectives: To describe a severe and bilateral neuritis with a positive MOG antibodies.

Results and Conclusions: Case report of a 19-year-old Brazilian woman who was positive for anti-MOG antibodies and negative for anti-aquaporin 4 antibodies.

The patient had one severe episode of bilateral optic neuritis. Magnetic resonance imaging brain is normal, atrophy and at optic nerve to retrochiasmatic contrast enhancement, and small lesions in cervical and dorsal spine. The patient did not have myelitis associated. Pulsotherapy was realized.

After 2 years the patient have a severe visual impairment and no one episode of demyelinating disease.

The case report supports the relationship between anti-MOG antibodies and MRI findings with one severe bilateral optic neuritis with atrophy and contrast enhancement and asymptomatic myelitis. Additional studies are needed to establish the clinical and radiological significance of anti-MOG antibodies. Funding: No funding or sponsorship was received for this research.

Keywords: Magnetic resonance imaging; Multiple sclerosis; MOG antibodies; Optic neuritis. 


\section{ORAL ABSTRACTS}

\section{OP01}

Neuromyelitis Optica Spectrum Disorders: Multicentric Study in Portugal

Ernestina Santos ${ }^{1,2}$; Ana Luísa Rocha ${ }^{3,4}$; Vanessa Olival ${ }^{1}$; Daniela Boleixa ${ }^{5}$; Daniela Ferro ${ }^{3,4}$; Raquel Samões ${ }^{1}$; Ana Paula Sousa ${ }^{6}$; Sónia Figueiroa ${ }^{7}$; Paula Carneiro ${ }^{8}$; Esmeralda $\mathrm{Neves}^{8}$; Teresa Mendonça ${ }^{3}$; Pedro Abreu ${ }^{3,4}$; Joana Guimarães $^{3,4}$; Ana Martins Silva ${ }^{1,2}$; Maria Isabel Leite ${ }^{9}$; Maria José Sá ${ }^{\prime, 10}$

${ }^{1}$ Department of Neurology, Hospital Santo António, Centro Hospitalar Universitario do Porto; ${ }^{2}$ Biomedical Research Multidisciplinary Unit, Instituto Ciências Biomédicas Abel Salazar, Universidade do Porto; ${ }^{3}$ Department of Neurology, São João University Hospital Center, Porto, Portugal; ${ }^{4}$ Department of Clinical Neurosciences and Mental Health, Faculty of Medicine, University of Porto, Portugal; ${ }^{5}$ Research Department, Hospital Santo António, Centro Hospitalar Universitario do Porto; ${ }^{6}$ Department of Neurophysiology, Hospital Santo António, Centro Hospitalar Universitario do Porto; ${ }^{7}$ Department of Neuropediatrics, Centro MaternoInfantil Albino Aroso, Centro Hospitalar Universitario do Porto; ${ }^{8}$ Department of Immunology, Centro Hospitalar Universitario do Porto; ${ }^{9}$ Nuffield Department of Clinical Neurosciences, University of Oxford, UK; ${ }^{10}$ Faculty of Health Sciences, University Fernando Pessoa, Porto, Portugal

Introduction: Neuromyelitis optica spectrum disorder (NMOSD) is a rare immune mediated disease of the central nervous system. We are conducting a national multicentric study to identify and characterize our NMOSD population.

Objectives: To present preliminary data from the two centers proposing this study.

Methodology: We identified 58 NMOSD patients from our clinical databases. Informed consent was signed, and anonymized data inserted an electronic database. Serological studies (AQP4 and MOG antibodies) were completed in 47 patients. Eleven patients tested only for anti-AQP4 were not included.

Results and Conclusions: Results: Twenty-four patients had AQP4 antibodies, 17 had MOG antibodies; 6 were double seronegative. In the AQP4 seropositive group the female/male ratio was $5: 1$, average age at onset was 39.8 years (4-87). The first manifestation was in 9 optic neuritis (ON), 10 myelitis, 2 brainstem syndrome, 1 area postrema syndrome, 1 narcolepsia and 1 with simultaneous myelitis, brainstem and area postrema syndrome. Annualized relapse rate was 0.48 . The treatments in the acute phase were steroids (24), IVIG (10) and plasma exchange (5). Chronic treatments were azathioprine (17), MMF (2), MTX (2), cyclophosphamide (2) and rituximab (4). MOG antibody positive patients were 9 women and 8 men (ratio 1.1:1), average age at onset 37.6 years (12-66). First manifestation was in 5 ON, 8 myelitis, $3 \mathrm{ON}$ and myelitis and 1 supratentorial symptoms. Seven had monophasic disease, annualized relapse rate was 0.49 . In the acute phase were treated with steroids (16), IVIG (1) and plasma exchange (2). Chronic treatments were azathioprine (6) and rituximab (2). The double seronegative subgroup had 3 women and 3 men, average age at onset of 35 years (22-53). First manifestation was $1 \mathrm{ON}, 3$ myelitis, 1 myelitis and supratentorial symptoms and 1 supratentorial symptoms. They were treated with steroids 5, IVIG 3, plasma exchange 2, azathioprine 2 and MTX 1 . Another autoimmune disorder was identified in 12, 2 and 1 patients in the AQP4, MOG and double seronegative subgroups, respectively.

Discussion: This preliminary study constitutes the foundation for a larger and more comprehensive clinical and serological study at the national level, to identify and characterise all NMOSD patients and ultimately improve their care.

Funding: This research was sponsored by Roche. Keywords: Neuromyelitis optica spectrum disorders

\section{OPO2}

Pilot Study of Telemedicine in Multiple Sclerosis to Evaluate the Effectiveness of a Telecommunication System for the Detection of the Clinical Activity of the Disease in the Number of Relapses with Respect to the Standard Clinical Practice. Telem [8]

Anna Gil-S²; Beatriz Pardiñas ${ }^{3}$; José Vicente Hervás ${ }^{3}$; Lara Nogueras $^{1}$; Hugo Gonzalo ${ }^{3}$; Cristina González-Mingot ${ }^{3}$; Marc Canudes ${ }^{2}$; Silvia Peralta ${ }^{4}$; Maria José Solana ${ }^{3}$; Petya Valcheva $^{2}$; Luis Brieva ${ }^{3}$

${ }^{1}$ Universitat de Lleida; ${ }^{2}$ Institut de Recerca Biomèdica de Lleida; ${ }^{3}$ Hospital Universitari Arnau de Vilanova de Lleida; ${ }^{4}$ Fundació Esclerosis Múltiple de Lleida

Introduction: Telemedicine is a method to provide health care services at a distant site.

Objectives: The aim of this study was to measure the ability of telemedicine to detect relapses in patients with multiple sclerosis (MS). The second aim was to observe the difference between patients with cognitive impairment and cognitive preservation.

Methodology: 130 patients under treatment with firstline drugs for MS who had the computer skills to access a web platform created by this study were recruited. The patients had to answer surveys about their clinical status and were randomized in two groups: survey (intervention) and non-survey (control). Also, three visits were made during the study: the basal visit, visit 6 and visit 12 months. All patients fulfilled questionnaires of mood, health, satisfaction with health services and adherence to treatment. They performed the Multiple Sclerosis Functional Composite (MSFC), and the Expanded 
Disability Status Scale (EDSS). The cognitive status was estimated using two tests for cognitive ability measurement: the Symbol Digit Modalities Test (SDMT) and the Paced Auditory Serial Addition Test (PASAT) and a subsequent classification was made: cognitive impairment/suspected impairment/cognitive preservation.

Results and Conclusions: The sensitivity of the platform survey was $100 \%$ and the specificity $96.5 \%$. Both groups were equal in all the analyzed features, with the exception of the EDSS, which was significantly higher in the intervention group than in the control group (mean 0 vs. 1). There were no more relapses in the survey/intervention group (average 0 vs. 0). Patients with cognitive impairment had a worse mood at the beginning of the study and were less satisfied with the health services received than the cognitively preserved, but they showed the same adherence as them.

Telemedicine is a useful tool in detecting relapses in MS patients and cognition is an important factor in the health and mood of patients with multiple sclerosis.

Funding: This research was sponsored by Novartis pharmaceuticals.

Keywords: Telemedicine; Cognition

\section{OPO3}

Associations Between Coffee Intake, Clinical and Demographic Characteristics in a Cohort Of Patients with Multiple Sclerosis [9]

Sara Duarte ${ }^{1}$; Denis Gabriel ${ }^{1}$; Inês Moreira ${ }^{2}$; Daniela Boleixa $^{3}$; Raquel Samões ${ }^{1}$; Ana Paula Sousa ${ }^{1}$; Andreia Bettencourt $^{3,4}$; Ana Martins Da Silva ${ }^{1,4}$; Ernestina Santos ${ }^{1,4}$

${ }^{1}$ Department of Neurology, Hospital de Santo António, Centro Hospitalar do Porto, Portugal; ${ }^{2}$ Laboratory of Neurobiology of Human Behavior, Hospital de Santo António, Centro Hospitalar do Porto, Portugal; ${ }^{3}$ Immunogenetics Laboratory, ICBAS, University of Porto, Portugal; ${ }^{4}$ Unit for Multidisciplinary Research in Biomedicine, ICBAS, University of Porto, Portugal

Introduction: Lifestyle factors are key contributors to the risk of multiple sclerosis (MS). Previous studies on caffeine and risk of MS have yielded different results; one study suggests a positive effect on disease progression. Objectives: Examine associations between coffee consumption, demographic and clinic characteristics in MS patients.

Methodology: Questionnaires were applied to 126 MS patients that attended neuroimmunology consultation between January-November/2017. MS severity was evaluated by EDSS, 9-HPT, T25-FW and SDMT. High coffee intake was considered $\geq 4$ cups/day; lifetime consumption was calculated multiplying espressos per day by years of consumption. Fisher exact test, Mann-Whitney test and Pearson correlation were used, as appropriated.

Results and Conclusions: Our cohort had an average age of 45.3 years and 76 patients (60.3\%) were women. Mean age of MS onset was 29.7 years and mean duration of disease 15.6 years. Seventy-nine percent had relapsing-remitting MS; $50 \%$ had EDSS $0-3.5,35.7 \%$ EDSS $=3.5-6.0$, and $14.3 \%$ EDSS $\geq 6.0$. A third were current or past smokers. Regular coffee intake was observed for $78.6 \%$ of the patients, and high coffee intake was present in $17.2 \%$. Men had more high coffee intake ( $29 \%$ vs. $10.3 \%, p=0.03)$ but the total coffee consumption was not different between genders $(p=0.48)$. High coffee intake and total coffee consumption was associated with higher smoking habits (3.85 vs. 0 pack-years, $p=0.01$; $r=0.29, p<0.001$ ).

No significant association was found between EDSS and high coffee intake or total coffee consumption (3 vs. $3.5, p=0.79 ; r=0.06, p=0.48$ ). Also, we did not find any differences regarding coffee intake and performance in 9-HPT, T25-FW and SDMT. Gender and smoking can be possible cofounders, but were not associated with the results in these tests nor with EDSS. In our cohort, coffee consumption does not seem to have a role in the progression of disability. Further studies are required to access this association and to evaluate the mechanisms by which coffee may be acting in MS.

Funding: No funding or sponsorship was received for this research.

Keywords: Multiple sclerosis; Coffee intake; Disability

\section{OPO4}

Targeting S100B Prevents in Vivo Multiple SclerosisLike Pathology

Catarina Barros ${ }^{1}$; Andreia Barateiro ${ }^{1}$; Raquel Freitas ${ }^{2}$; Dora Brites $^{1,3}$; Luis Graça ${ }^{2}$; Adelaide Fernandes ${ }^{1,3}$

${ }^{1}$ Neuron-Glia Biology in Health and Disease, Research Institute for Medicines (iMed.ULisboa), Faculty of Pharmacy, Universidade de Lisboa, Portugal; ${ }^{2}$ Instituto de Medicina Molecular, Faculdade de Medicina, Universidade de Lisboa, Portugal; ${ }^{3}$ Department of Biochemistry and Human Biology, Faculty of Pharmacy, Universidade de Lisboa, Portugal

Introduction: S100B, a small inflammatory molecule, has been extensively studied in neurodegenerative and neuroinflammatory disorders, namely in 
Multiple Sclerosis (MS). We found that S100B is present in the CSF and serum of MS patients and, recently, we associated high levels of S100B with demyelination and inflammatory processes. Using an ex vivo demyelinating model, blockade of S100B with pentamidine, a specific S100B-binding drug, showed promising results indicating this targeting as an emerging therapeutic strategy for MS.

Objectives: Here, we aimed to understand whether the targeting of S100B, through drug- modifying therapy, could ameliorate in vivo MS-like pathogenesis.

Methodology: Experimental autoimmune encephalomyelitis (EAE) was induced in female C57BL/6J wild-type mice divided in two groups: vehicle (saline) and treated (pentamidine, $4 \mathrm{mg} / \mathrm{kg}$ i.p.-daily). Then, we evaluated clinical score and body weight during 30-days of experiment. To evaluate CNS pathogenesis, we assessed the expression of specific markers of immature and mature oligodendrocytes, glial reactivity, and inflammation through immunohistochemistry and qRT-PCR.

Results and Conclusions: Using the in vivo animal model of MS, the EAE, we observed that pentamidine treatment was able to affect disease progression either delaying the appearance of the first clinical signs but also reducing severity and improving recovery. Moreover, we also observed that pentamidine could prevent demyelination and oligodendrogenesis impairment. Interestingly, pentamidine also reduced glial reactivity and consequently shifted from a proinflammatory environment to a more anti-inflammatory one as shown by the decrease of TNF- $\alpha$ and IL$1 \beta$ and increase of IL-10, respectively, corroborating the preventive properties of pentamidine.

Overall, our results indicate that $\mathrm{S100B}$ is involved in MS pathology and that, by using pentamidine, we could prevent the detrimental effects of this protein when in pathological levels. So, S100B inhibition may be a new therapeutic strategy with broaden efficacy to reduce MS-like pathogenesis and CNS damage.

Funding: This research was sponsored by GMISI-Merck to AF, and by FCT-UID/DTP/04138/2013 to iMed. ULisboa and SFRH/BPD/96794/2013 to AB.

Keywords: EAE; S100B; Demyelination; Neuroinflammation
OP05

Autologous Hematopoietic Stem Cell Transplantation in Multiple Sclerosis: Preliminary Results from a Tertiary Centre

Daniela Vieira ${ }^{1}$; Inês Correia ${ }^{1}$; Sónia Batista ${ }^{1}$; Carla Nunes ${ }^{1}$; Fernando Matias ${ }^{1}$; Isabel Campelo ${ }^{2}$; Carmo Macário ${ }^{1}$; Catarina Geraldes ${ }^{3}$; Lívia Sousa ${ }^{1}$

${ }^{1}$ Serviço de Neurologia, Centro Hospitalar e Universitário de Coimbra; ${ }^{2}$ Serviços Farmacêuticos, Centro Hospitalar e Universitário de Coimbra; ${ }^{3}$ Serviço de Hematologia Clínica, Centro Hospitalar e Universitário de Coimbra

Introduction: Autologous hematopoietic stem cell transplantation (AHSCT) has been proposed for aggressive multiple sclerosis (MS) when other treatments fail. As a rescue treatment, its goals are to promote neurological stability and possibly to improve neurological function.

Objectives: To evaluate clinical features and early outcomes after AHSCT for treatment of MS.

Methodology: We performed a retrospective study that included all the MS patients submitted to AHSCT in our center, and collected clinical data from medical records.

Results and Conclusions: Six patients were included (3 females), of which 4 patients had SPMS and 2 patients had RRMS. These patients had a mean age of 38.5 years (32-42) and 11.2 years of disease duration (7-18). All the patients received multiple previous treatments including approved and off-label immunosuppressive disease-modifying treatments $(9$ in one patient, 7 in one patient, 6 in one patient, 4 in one patient and 3 in two patients). All of them had severe motor disability including hand function impairment and were worsening. Median EDSS score prior to AHSCT was 6.5 (6.0-7.5), but cognitive function was preserved. They received the BEAM conditioning regimen (carmustine, etoposide, cytarabine and melphalan) and had a mean inpatient period in hematopoietic transplantation unit of 35.7 days. The mean follow-up after AHSCT was 18.5 months (3-56). After the procedure, the EDSS score improved in three patients $(0.5-1)$ and stabilized in the others who achieved AHSCT in the last year. The overall most significant neurologic benefit was clinical stabilization with hand function improvement and the ability to get some walk independence. MRI was available in two patients and showed no lesion load increase. The most common early complications were gastrointestinal toxicity $(n=6)$ and febrile neutropenia $(n=4)$.

AHSCT in our small number of patients, who had aggressive MS irresponsive to several disease-modifying treatments, was effective in achieving disability stabilization with improvement in some cases. 
Funding: No funding or sponsorship was received for this research.

Keywords: Autologous stem cell transplantation; Aggressive multiple sclerosis; Motor disability; BEAM conditioning regimen.

\section{OP06}

Infectious Diseases Department Interventions in Neurological Immunosuppressed Patients

Maria João Gonçalves ${ }^{1}$; José Carvalho ${ }^{2}$; Isabel Furtado ${ }^{1}$; Ana Horta $^{1,3}$; Sandra Xará1; Miguel Araújo Abreu ${ }^{1,4}$; Ernestina Santos $^{4,5}$; Ana Martins Da Silva ${ }^{4,5}$; Rui Sarmento e Castro ${ }^{1,4}$

${ }^{1}$ Infectious Diseases Department, Centro Hospitalar e Universitário do Porto; ${ }^{2}$ Internal Medicine Department, Unidade Local de Saúde do Alto Minho; ${ }^{3}$ Escola de Medicina da Universidade do Minho; ${ }^{4}$ Instituto de Ciências Biomédicas Abel Salazar; ${ }^{5}$ Neurology Department, Centro Hospitalar e Universitário do Porto

Introduction: In recent years there is an increased use of immunotherapies in inflammatory neurological diseases. These drugs are highly effective in controlling these diseases, however, they lead to an increased risk of infections.

Objectives: To describe the epidemiological, clinical and analytical characteristics of neurological patients submitted to immunomodulation/immunosuppression, followed in consultation for screening, prevention, monitoring and treatment of infections.

Methodology: Retrospective study of patients sent by the Neurology Department to the Infectious Diseases outpatient clinic to evaluate the infectious risk, from February 2017 to December 2018.

Results and Conclusions: Forty-one patients were included: 24 females, average age 45.4 years [19-78 \pm 12.3 ]. The mean duration of neurological disease was 7.5 years $[0.5-22 \pm 5.6]$. Thirty-four patients had multiple sclerosis, three myasthenia gravis, three CNS vasculitis and one chronic inflammatory demyelinating polyneuropathy. Previous immunosuppression was described in 23 (8 biological).

The plan was to begin (18), switch (15) or maintain (8) the immunosuppression; 25 with biological therapy, 15 with other immunosuppressants and one pending decision.

All patients were screened and showed to be negative for Human Immunodeficiency virus, syphilis, chronic hepatitis $\mathrm{B}(\mathrm{HBV})$ or $\mathrm{C}$ virus, with no diagnostics. Epstein-Barr and Zoster Virus IgG was universal.

Twelve patients were diagnosed with latent tuberculosis, all started isoniazid $300 \mathrm{mg} /$ day, three discontinued due to toxicity ( 2 switched to rifampicin).

The influenza and pneumococcal conjugate polysaccharide vaccine was suggested to all patients (2 refused). The $\mathrm{HBV}$ and hepatitis A vaccination was proposed to 21 and 12 non- immune patients, respectively.

Trimethoprim-sulfamethoxazole and acyclovir were instituted in four and two patients, respectively. No serious infection or death was observed during follow-up.

Through the strategies implemented (treatment of latent tuberculosis, immunizations and prophylaxis) we aim to minimize the infectious risk.

Multidisciplinarity and the development of an individualized plan according the patient characteristics and immunosuppression, is essential for the safe use of these drugs.

Funding: No funding or sponsorship was received for this research.

Keywords: Infectious diseases; Infectious risk

\section{ACKNOWLEDGEMENTS}

Funding. The studies referred to in the abstracts in this supplement may have been funded by sponsors, grants or institutions. The article processing charges for the publication of this supplement was funded by Roche. The supplement funder had no involvement in the writing or research, unless indicated for an abstract, and no involvement in the Scientific Committee's review procedure.

Authorship. All named authors meet the International Committee of Medical Journal Editors (ICMJE) criteria for authorship for their abstracts, take responsibility for the integrity of the work as a whole, and have given their approval for this version to be published.

Disclosures. Authors of abstracts may have relevant disclosures, which are not published here as this is a compilation of the abstracts only.

Compliance with Ethics Guidelines. The Supplement includes abstracts, not full original research articles, so that compliance with ethics is not reported. The Criteria for abstract selection were: Submitted abstracts were evaluated by at least 2 members of the scientific committee based on originality, the relevance of findings, the soundness of the content and the 
structural quality. The supplement was then peer reviewed by an Editorial Board Member of Neurology and Therapy, independent of the meeting's abstract submission process.

Open Access. This article is distributed under the terms of the Creative Commons Attribution-NonCommercial 4.0 International License (http://creativecommons.org/licenses/ by-nc/4.0/), which permits any noncommercial use, distribution, and reproduction in any medium, provided you give appropriate credit to the original author(s) and the source, provide a link to the Creative Commons license, and indicate if changes were made.

\section{REFERENCES}

1. EPIO02, Martins R, Albuquerque C. Quality of life of the person with multiple sclerosis. Encore abstract presented at the 3rd International Porto Congress of Multiple Sclerosis, Porto, Portugal, 27-28 February 2015. Sinapse, Volume 15, No.1, May 2015, p.165. Reproduced with permission.

2. EPI010, Schippling S, Sormani MP, De Stefano N, Giovannoni G, Galazka A, Keller B, Alexandri N. CLARITY: an analysis of severity and frequency of relapses in patients with relapsing-remitting multiple sclerosis treated with cladribine tablets or placebo. Encore abstract presented at the 34th Congress of the European Committee for Treatment and Research in Multiple Sclerosis (ECTRIMS) Berlin, Germany, 10-12 October 2018. http://www.professionalabstracts.com/ ectrims2018/iplanner/\#/presentation/1088. (c) The Author(s).

3. EPI011, Giovannoni G, Montalban X, Rammohan K, Cook S, Comi G, Rieckmann P, Soelberg-Sorensen P, Vermersch P, Dangond F, King J, Roy S, Damian D. P28 efficacy of cladribine tablets in patients with highly active relapsing-remitting multiple sclerosis: analysis of pooled double-blind data from the CLARITY and ONWARD studies. Abstract presented at The Australian \& New Zealand Association of Neurologists (ANZAN) 2018 ASM May 29-June 1, J Neurol Neurosurg Psychiatry 2018;89:A27-A28. Encore abstract presented at the Annual Congress 2018 of the Swiss Neurological Society, Vaud, Switzerland, Sep 27-28, Clinical \& Translational Neuroscience 2018: 2(Supplement) 1S-34S. https://doi.org/ 10.1177/2514183X18802121 Reproduced with Permission.
4. EPII016, Cook S, Giovannoni G, Vermersch P, Soelberg-Sorensen P, Keller B, Jack D. Lymphopenia rates in CLARITY/CLARITY Extension are unrelated to disease activity at baseline. Encore abstract presented at the 34th Congress of the European Committee for Treatment and Research in Multiple Sclerosis (ECTRIMS) Berlin, Germany, 10-12 October 2018. http://www.professionalabstracts.com/ectrims2018/ iplanner/\#/presentation/1431. (C) The Author(s).

5. EPIII024, Sormani MP, Freedman MS, Aldridge J, Marhardt K, De Stefano N. Disease activity as assessed by the MAGNIMS score predicts long-term clinical disease activity (CDA)-free status and disability progression in patients treated with subcutaneous interferon beta-1a. Encore Abstract: 4th Congress of the European Academy of Neurology, Lisbon, Portugal, June 2018, EAN 2019 ePresentation Sessions. Eur J Neurol 25(2):277-573. https://doi.org/10.1111/ene. 13700. Reproduced with permission.

6. EPIII029, Brandão Nascimento AC, Araujo e Araujo AR, Meneguette NS, Papais Alvarenga M, Vasconcelos CCF, Bento CAM, Rafful PP, Brandao LA, Papais Alvarenga RM. Magnetic resonance imaging findings at optic neuritis of recurrent opticspinal phenotype, multiple sclerosis in a cohort of Brazilian patients from Rio de Janeiro. Abstract accepted to be held at the European Congress of Radiology, Vienna, Austria, February 27-March 3, 2019. Insights into Imaging. 2019;10(Suppl 1):22 p.S301. https://insightsimaging. springeropen.com/track/pdf/10.1186/s13244-0190713-y. (c) The Author(s).

7. EPIV034, das Neves SP, Serre-Miranda C, Nobrega C, Roque S, Cerqueira JJ, Correia-Neves M, Marques F. Immune thymic profile of the MOG-induced experimental autoimmune encephalomyelitis mouse model. Front Immunol. 2018;9:2335. 10.3389/ fimmu.2018.02335. https://www.frontiersin.org/ articles/10.3389/fimmu.2018.02335/full? (c) The Author(s).

8. OP02, Gil A, et al. Pilot Study of Telemedicine in multiple sclerosis to evaluate the effectiveness of a telecommunication system for the detection of the clinical activity of the disease in the number of relapses with respect to the standard clinical practice. TELEM. E-poster presented at the 34th Congress of the European Committee for Treatment and Research in Multiple Sclerosis (ECTRIMS) Berlin, 10-12 October 2018. https://www.ectrims-congress.eu/2018/ abstracts/eposters-only.html. (C) The Author(s).

9. OP03, Duarte S, et al. Coffee intake and multiple sclerosis disability. E-poster presented at the 34th Congress of the European Committee for Treatment and Research in Multiple Sclerosis (ECTRIMS) Berlin, 10-12 October 2018. https://www.ectrims-congress. eu/2018/abstracts/eposters-only.html. (c) The Author(s). 